\title{
On the synergy between physical and virtual sheet metal testing: calibration of anisotropic yield functions using a microstructure-based plasticity model
}

\author{
Coppieters S. · Hakoyama T. · Eyckens \\ P. · Nakano H. · Van Bael A. . \\ Debruyne D. . Kuwabara T.
}

Received: date / Accepted: date

\begin{abstract}
This paper scrutinizes in detail the synergy between physical and virtual testing of the mechanical response of sheet metal. In this context, physical testing refers to the usage of physical samples onto which mechanical tests are conducted, while virtual testing refers to multi-scale plasticity simulations onto a model representation of the metallic microstructure derived from microstructural measurement.

An extensive experimental campaign was conducted to capture the plastic material response of mild steel sheet in the first quadrant of the biaxial stress space, i.e. for tensile stresses along the Rolling Direction (RD) and Transverse Direction (TD). The experimental data was acquired using state-of-the-art stress-controlled material tests enabling to probe the material up to large
\end{abstract}

\footnotetext{
Coppieters S.

Department of Materials Engineering, KU Leuven, Campus Ghent, Gebroeders De Smetstraat 1, 9000 Ghent, Belgium

Tel.: +3293316672

E-mail: sam.coppieters@kuleuven.be

Hakoyama T.

G-CADET, Department of Mechanical Engineering, Gifu University, Japan

Eyckens P.

Department of Materials Engineering, KU Leuven, Belgium

Nakano H.

Graduate School, Department of Mechanical Systems Engineering, Tokyo University of Agriculture and Technology, Japan

Van Bael A.

Department of Materials Engineering, KU Leuven, Belgium

Debruyne D.

Department of Materials Engineering, KU Leuven, Campus Ghent, Gebroeders De Smetstraat 1, 9000 Ghent, Belgium

Kuwabara T.

Division of Advanced Mechanical Systems Engineering, Institute of Engineering, Tokyo University of Agriculture and Technology, Japan
} 
plastic strains in the first quadrant of stress space. Identical stress paths were simulated using the Virtual Experimentation Framework (VEF) software suite adopting the ALAMEL multi-scale plasticity model. The ALAMEL model was calibrated solely on the basis of the initial crystallographic texture of the material and a reference hardening curve obtained through a uniaxial tensile test in the rolling direction. The predictive accuracy of the ALAMEL model to reproduce the experimentally acquired material response has been thoroughly assessed. To avoid any bias in the assessment due to extrapolation of the material behavior, predictions were limited to the pre-necking regime of the material. The predictions of the VEF show good agreement with the physical test results.

Subsequently, the experimental and virtual test data were used to calibrate Hill's quadratic yield criterion and the Yld2000-2d yield function. The calibration accuracy of these yield criteria is thoroughly assessed by comparing theoretical predictions and experimental data. In addition, the calibrated yield functions are used to simulate a hydraulic bulge test and the results are compared with experimental observations. It is shown that the adopted virtual material testing procedure has reached a sufficient level of maturity to potentially serve as a viable alternative for physical material testing of steel sheet.

Keywords Virtual material testing - Mechanical testing - Sheet metal · Anisotropic yield function

\section{Introduction}

Finite Element (FE) modelling is extensively used to cope with growing performance demands (e.g. weight reduction) in the mechanical design of products and structures. The application of complex sheet metal forming processes to emerging alloys poses new manufacturing challenges which can be tackled with the aid of FE techniques. Most commercially available FE codes use macroscopic anisotropic models to capture the plastic response of sheet metal. Large simulation accuracy in sheet forming operation can be achieved by taking the directionality of mechanical properties of sheet metal into account through advanced phenomenological anisotropic yield functions. The flexibility of such macroscopic models to accurately describe the plastic anisotropic response of sheet metal comes at the expense of increased experimental effort. Indeed, the complexity of the continuum model usually scales with the amount of unknown model parameters, and, consequently, also with the effort (e.g experimental, computational, etc.) to identify them. From an industrial point of view, these continuum models are computationally efficient but their application is often impeded by the associated experimental calibration effort. Moreover, industrial practice generally aims at a minimum number of experiments, often leading to limited prediction quality.

Obviously, the predictive accuracy of a material model also depends on the quality of the experimental data used for calibration purposes. It makes sense, 
for example, to calibrate the model using carefully selected experimental data resembling (e.g. in terms of stress state(s)) the sheet forming process that needs to be simulated afterwards. In general, however, the identification of the governing model parameters relies on standard procedures limited to uniaxial and balanced biaxial tensile testing. Model parameters are often determined by minimizing the discrepancy between experimental and theoretical flow stresses and $r$-values through a cost function.

The most commonly adopted strategy to acquire the calibration data is to subject the sheet metal to physical experiments. Conventionally, sheet metal is subjected to stress-controlled testing (i.e. the stress state is precisely controlled in the gauge section of the specimen) at a constant rate of deformation. Uniaxial tensile experiments are easy to perform but it is well-known that biaxial tension experiments significantly enhance the calibration accuracy of advanced anisotropic yield functions [1]. Such experiments, however, entail a significant experimental effort and require dedicated test machines enabling to control a targeted stress path whilst maintaining a constant rate of deformation. Such methods, e.g. [2,3], are state-of-the art and extremely important to carefully verify concepts and theories [4]. Moreover, multi-axial stress-controlled experiments can be used to validate alternative identification strategies such as the Finite Element Model Updating (FEMU) method, Integrated Digital Image Correlation (IDIC) and the Virtual Fields Method (VFM). The latter methods can be employed to reduce the experimental work load to calibrate complex plasticity models by extracting more information from a single, carefully designed experiment. The FEMU method is straightforward in the sense that it relies on the minimization of the discrepancy between an experimentally measured and a numerically computed response (e.g. strain fields).

Multiple studies, e.g.[5-7], report on the identification of anisotropic yield functions through FEMU and full-field measurements. IDIC [8] is essentially based on global DIC $[9,10]$ and has for example been used to identify Hill's 48 yield criterion [11]. The VFM [12] offers a computationally efficient procedure to identify material behavior from full field measurements. To do so, the VFM framework relies on the minimization of the difference between the virtual internal and the virtual external work. The nonlinear VFM has been successfully applied $[13,14]$ to identify constitutive parameters of anisotropic yield loci. Although it has been demonstrated that FEMU, IDIC and VFM have the potential to reduce the amount of experimental effort in calibrating advanced continuum plasticity models, a number of issues require more research. For example, a systematic procedure to design the optimal specimen geometry [15] preferably as a function of a specific material state is required. In addition, the influence of experimental errors in acquiring full-field data [16] on the identified parameters needs to be investigated thoroughly. Finally, verification of alternative methods should be pursued by consistent comparison with state-of-the-art stress-controlled sheet metal tests.

Another possibility to reduce the experimental work load associated with the calibration of anisotropic yield functions is to resort to so-called virtual material testing. Virtual testing refers to multi-scale plasticity simulations 
onto a model representation of the metallic microstructure derived from microstructural measurement. A virtual laboratory $[17,18]$ is then used to conduct stress-driven crystal plasticity (CP) simulations substituting the physical stress-controlled experiments. Several authors embarked on the calibration of macroscopic anisotropic yield functions for sheet metal using data generated by crystal plasticity models [4,19-24,17,25,26,18]. Barlat et al.[4] used a ViscoPlastic Self-Consistent (VPSC) model to generate data characterizing the outof-plane properties of an aluminium alloy sheet sample. Conventional testing data obtained from tensile tests, a hydraulic bulge test and a disk compression test supplemented with the virtual test data were used to calibrate Yld200418p. Plunkett et al. [19] combined experimental data with virtual data (generated using a VPSC model) to calibrate a macroscopic yield function capable of capturing differential hardening. Grytten et al. [20] scrutinized the capability of calibrating Yld2004-18p based on mixed experimental-virtual and fully virtual data. The FC Taylor model was used to generate the virtual data and the authors concluded that a more advanced $\mathrm{CP}$ model is required to arrive at a sufficiently accurate calibration procedure. Hammami et al. [27] showed that the Multisite model provides an accurate anisotropy prediction for Ti6Al4V under tensile stress states. The authors concluded that the Multisite model can be used to limit the experimental effort associated with the calibration of advanced anisotropic yield functions such as CPB06. Zhang et al. [26] concluded that the predictive accuracy of the FC Taylor, VPSC and ALAMEL models is insufficient to capture the anisotropic response of AA3103 sheet, and, as a consequence, deemed inadequate to calibrate Yld2004-18p. Gawad et al.[17] employed the ALAMEL model to fully calibrate the BBC2008 yield function to capture the plastic anisotropy of an industrial-grade aluminum alloy AA6016. The authors showed through cup drawing simulations that BBC2008 calibrated using virtual data can yield more accurate results than using conventional mechanical testing. Yamanaka et al.[25] employed the CPFE method to calibrate Yld2000-2d for 5182 aluminum alloy sheet. The authors concluded that virtual testing yields an equivalent calibration accuracy compared to the physical testing. Zhang et al.[18] presented a framework for a virtual test lab to calibrate phenomenological anisotropic yield functions. The methodology was applied to an AA3104 alumium alloy and the authors showed that the virtual laboratory can substitute physical, uniaxial tensile tests. It is clear from these studies that virtual material testing is a promising approach for efficient calibration of advanced anisotropic yield functions.

The key point is that CP models enable to acquire virtual test data for specific stress states provided that the crystallographic texture and, in some cases, statistical information about grain morphology, is available. Stress states which are difficult to probe experimentally can be substituted by their virtual counterpart. The latter approach will potentially gain importance since improved anisotropic yield functions will likely require more complicated experiments. Embarking on full virtual calibration requires a profound experimental validation of the adopted CP model. In essence, the CP model must be able to reproduce the material response during stress-controlled physical testing. 
In this study, the focus is on the synergy between physical and virtual testing of sheet metal using the ALAMEL model. To guarantee a high-resolution assessment of the $\mathrm{CP}$ predictions, the research efforts are confined to the first quadrant of stress space which is probed by 9 proportional stress paths. Additionally, to avoid any uncertainty with respect to extrapolation of material behavior [28], the assessment is solely based on experimental data in the prenecking region. First, the experimental stress-strain response is directly compared with the response obtained through the ALAMEL model. The experimentally observed and virtually predicted plastic anisotropy are thoroughly assessed. Next, both experimental and virtual data are used to calibrate Hill's 48 yield criterion and the Yld2000-2d yield function. The calibration accuracy is assessed by comparing theoretical predictions of the anisotropic yield functions with the physically acquired data. Finally, the experimentally and the virtually calibrated yield functions are embedded in FE models to simulate a hydraulic bulge test, followed by detailed experimental validations.

\section{Material Testing}

\subsection{Physical Testing}

An industrial cold-rolled steel sheet (deep-drawing quality and designated SPCE in Japanese Industry Standard, JIS) was chosen as test material in this study. The steel sheet has a nominal thickness of $1.2 \mathrm{~mm}$ and its chemical composition (mass\%) can be found in table 1. All material tests reported in this section comply with the following characteristics:

1. the Rolling Direction (RD) and the Transverse Direction (TD) are aligned with the $x$ and $y$ axis, respectively.

2. the reference plastic strain rate was kept constant to approximately $5 \times 10^{-4}$ $\mathrm{s}^{-1}$.

3. the equivalent plastic strain in the experiment is limited to the maximum uniform strain measured in a tensile test in the RD, see Table 2 .

4. 2 tests for each type of experiment.

Standard tensile tests (JIS 13 B-type) were conducted to determine the work hardening properties, the maximum uniform strain $\epsilon_{\max }$ and the $r$-values under $0^{\circ}, 45^{\circ}$, and $90^{\circ}$ with respect to the rolling direction (RD). The values can be found in Table 2. The experimental true stress-plastic strain curves up to $\epsilon_{\max }$ are shown in Figures 1 (a),(d) and (g). Swift's hardening law was used to realize the excellent fit for the RD (see Figure 1(a)).

The characterization was confined to the first quadrant of stress space and the material was subjected to 7 linear stress paths (true stress ratios $\sigma_{x}: \sigma_{y}=4: 1,2: 1,4: 3,1: 1,3: 4,1: 2$ and 1:4) using two types of biaxial tensile tests, i.e. a biaxial tensile test and a tube expansion test. The experimental campaign is schematically shown in Fig.2 and more details can be found in [29]. The cruciform specimen proposed by Kuwabara et al. [2] was used to measure the 
Table 1: Chemical composition test material.

\begin{tabular}{ccccc}
\hline Designation & C & Mn & P & S \\
\hline SPCE & $\leq 0.08$ & $\leq 0.45$ & $\leq 0.03$ & $\leq 0.03$
\end{tabular}

Table 2: Swift's hardening law $\left(\sigma=K\left(\epsilon_{0}+\epsilon_{e q}^{p l}\right)^{n}\right)$ fitted in a strain range from $\epsilon_{e q}^{p l}=0.002$ up to the maximum uniform strain $\epsilon_{\max }$. The reported $r$-values are the measured values at an engineering strain $\epsilon_{\text {eng }}=0.10$ using gauge marks.

\begin{tabular}{lllllll}
\hline Tensile direction & $\sigma_{0.2}(\mathrm{MPa})$ & $K(\mathrm{MPa})$ & $\epsilon_{0}$ & $n$ & $r$ & $\epsilon_{\max }$ \\
\hline $\mathrm{RD}(\mathrm{x})$ & 153 & 564 & 0.0059 & 0.275 & 1.85 & 0.248 \\
$45^{\circ}$ & 161 & 558 & 0.0072 & 0.272 & 1.93 & 0.254 \\
$90^{\circ}(\mathrm{y})$ & 162 & 549 & 0.0080 & 0.272 & 2.82 & 0.259 \\
\hline
\end{tabular}

plastic material response in the moderately low strain range (i.e. $\epsilon_{0}^{p}<5 \%$ ) according to [30]. The arms of the cruciform were parallel to the RD and TD of the as-received material and the normal strain components $\left(\epsilon_{x}, \epsilon_{y}\right)$ were measured using uniaxial strain gauges (YFLA-2, Tokyo Sokki Kenkyujo Co.) at the optimal positions $[31,32]$. The slits in the arms of the cruciform specimen were obtained by laser cutting. More details concerning the biaxial testing apparatus and the testing method can be found in [2] and [30], respectively.

The Multiaxial Tube Expansion Test (MTET) [33,3] was used to probe plastic deformations equivalent to the maximum uniform strain $\epsilon_{\max }$ determined through a uniaxial tensile test in the RD. Two types of tubular specimens were used, type I and type II, respectively. Type I had the RD oriented along the axial direction of the tube and was used for testing under the conditions for which $\sigma_{x} \geq \sigma_{y}$. Type II had the RD in the circumferential direction and was used for stress states satisfying $\sigma_{x} \leq \sigma_{y}$. More details on the MTET, the testing machine and the feedback circuit for controlling the true stress paths can be found in [3]. The experimental true stress-plastic strain curves obtained through the biaxial tensile experiments are shown in Fig.3. 


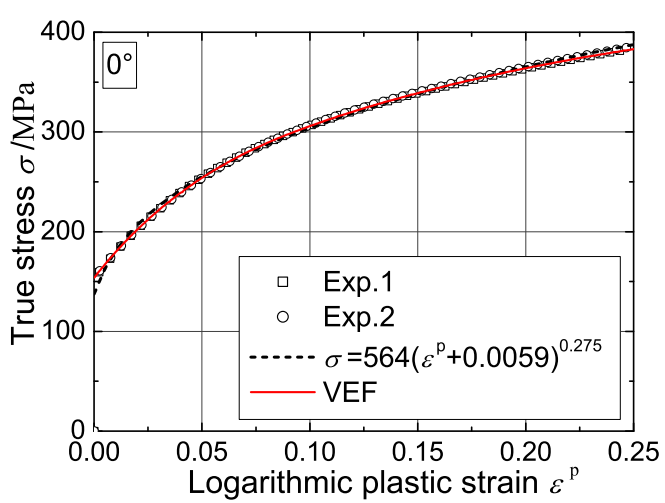

(a)

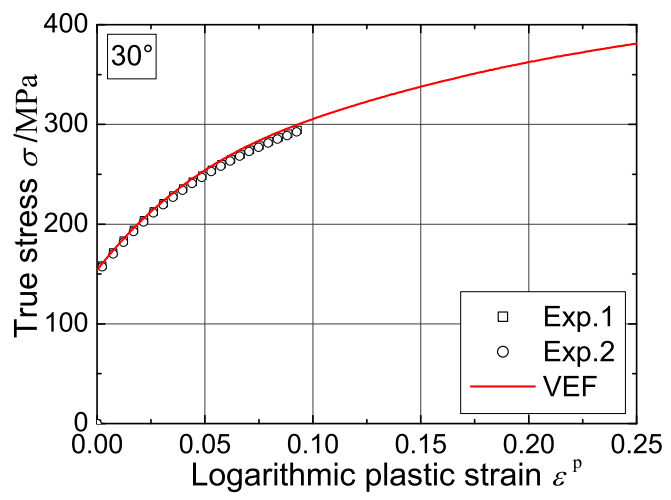

(c)

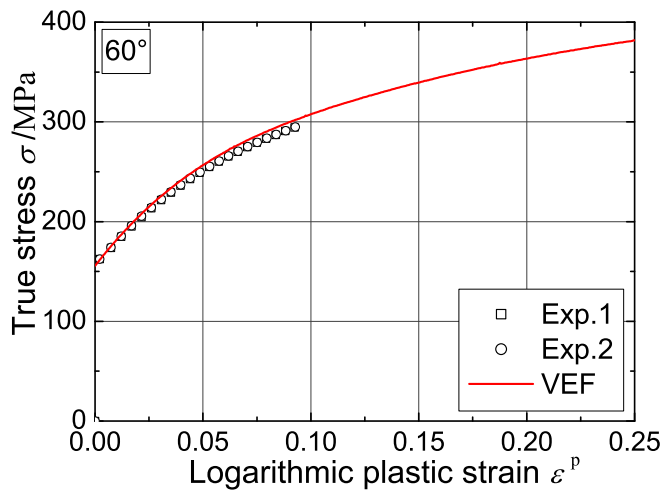

(e)

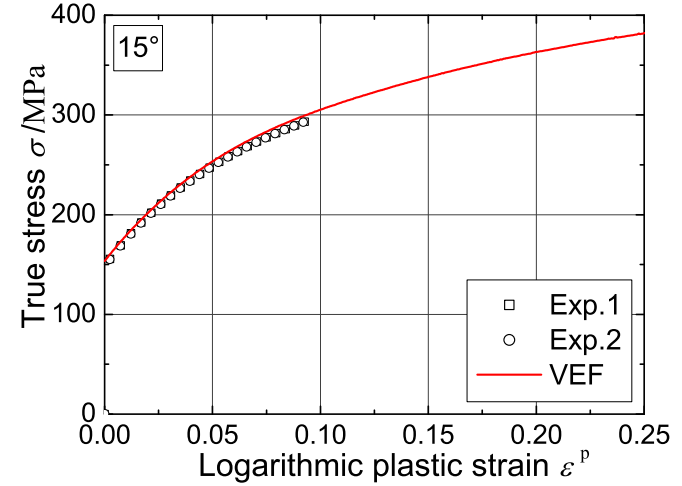

(b)

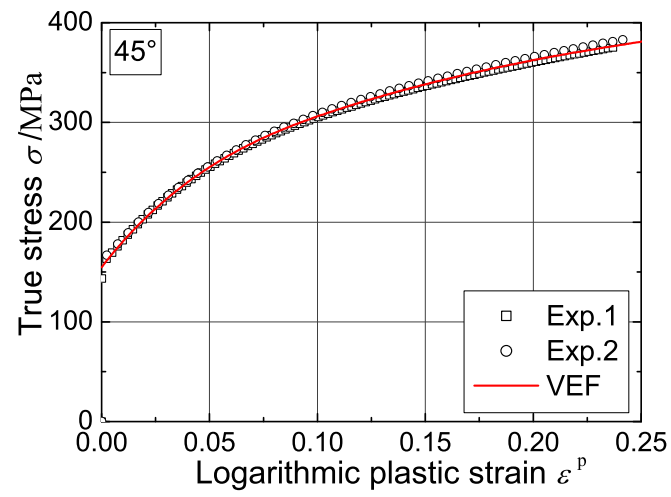

(d)

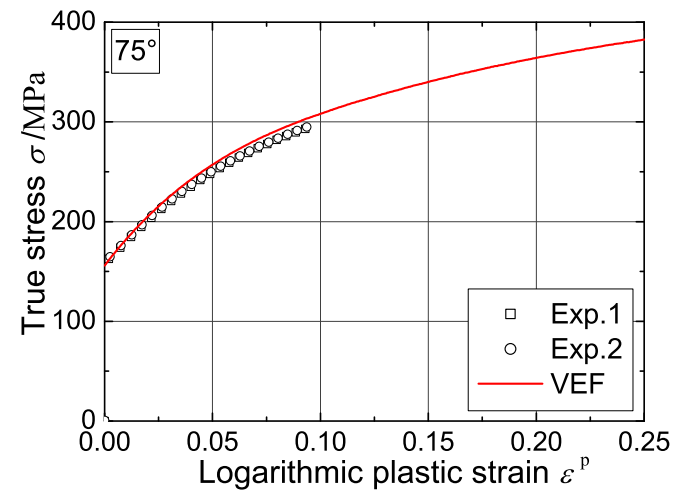

(f)

Fig. 1: Uniaxial stress-strain curves. (a) $0^{\circ}$, (b) $15^{\circ}$, (c) $30^{\circ}$, (d) $45^{\circ}$, (e) $60^{\circ}$, (f) $75^{\circ}$ 


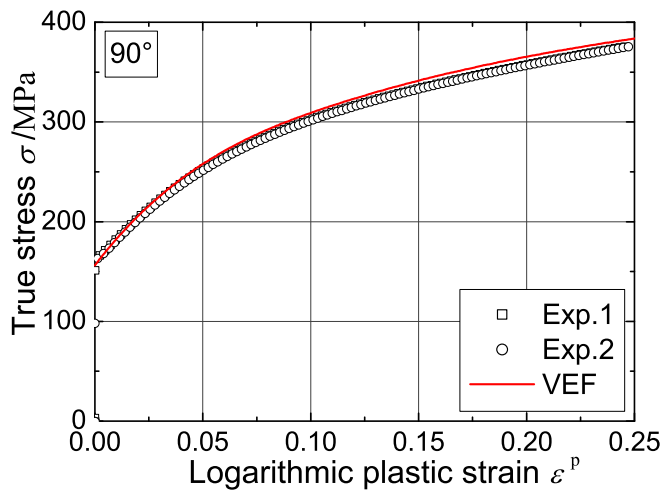

(g)

Fig. 1: (Cont'd) Uniaxial stress-strain curves. (g) $90^{\circ}$

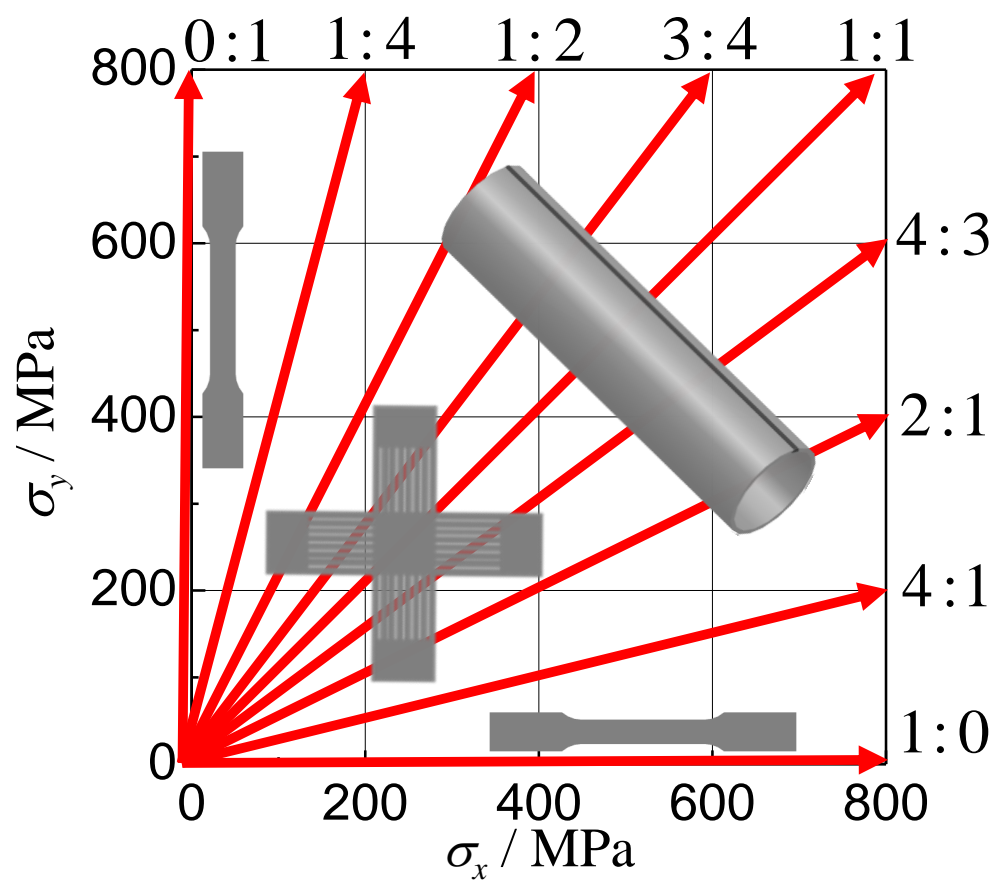

Fig. 2: Physical testing in the first quadrant of stress space: 9 linear stress paths. 


\subsection{Virtual Testing}

The procedure for virtual testing of the yield locus is based on the ALAMEL multi-scale model and is briefly outlined here. More details can be found in [34] (the original publication of the ALAMEL model) and [17] (procedure to employ the ALAMEL model for yield locus calibration). The multi-scale ALAMEL model makes use of a statistical representation of the microstructure whereby interaction between grains is accounted for. The microstructure is composed of a large number of independent microstructural units called clusters. A cluster consists of two neighbouring grains (crystals) of equal size. They are separated by a common, flat grain boundary with specific orientation. Upon imposing a plastic deformation on an ALAMEL cluster, particular slip systems in both constituting grains are activated in such a way that the plastic work that is associated with slip activation, is minimized for the cluster as a whole. The deformation inside each grain can hereby differ from the imposed deformation due to a simple shear deformation along the grain boundary (relaxation). As the relaxations are required to be opposite in both grains of the cluster, the average deformation inside the cluster equals the imposed deformation. It can be proven for this model that local stress equilibrium across the grain boundary is guaranteed under all conditions [34]. The anisotropic behavior of the aggregate of clusters follows from averaging the stress state in all grains, which is readily derived from the set of active slip systems and their associated Critical Resolved Shear Stresses (CRSS) that trigger plastic slip on those slip systems. The selection of grain orientations is derived from (crystallographic) texture measurement. For the material at hand, the texture was obtained by XRD pole figure measurements on samples at 3 different depths, namely at $0 \%, 25 \%$ and $50 \%$ of the sheet thickness. The texture gradient across the thickness was found to be weak. The pole figures at mid-thickness are presented in Figure 4(a). The Figure 4(b) shows the resulting Orientation Distribution Functions (ODF) calculated from the weighted average of measurements across the thickness (weighting factors of $0.25,0.5$ and 0.25 for the $0 \%, 25 \%$ and $50 \%$ samples, respectively).

It is seen from Figure 4(b) that the distribution of crystal orientations is concentrated in the so-called $\gamma$-fiber (a perfect gamma-fiber consists of all orientations with a $\{111\}$ crystal direction normal to the sheet plane), which appears horizontally in this section of Euler space (orientation space). This kind of $\gamma$-fibered texture is typical for cold rolled and recrystallized steel sheet. The detailed characteristics of the texture, such as its sharpness, are determined by the exact thermo-mechanical processing conditions and alloying content. These texture characteristics are responsible for the precise anisotropic plastic behavior, see e.g. [35]. In the present work, the virtual microstructure for the multi-scale ALAMEL simulations consists of a set of 5000 orientations (grains) that have been statistically sampled from the average ODF [36]. These grains have been placed at random in a set of 2500 clusters ( 2 grains per cluster). A grain boundary orientation is assigned at random to each cluster in order to statistically represent a microstructure with an equiaxed grain shape on aver- 
age, since this is the typical grain morphology in fully recrystallized condition as is the material under investigation. For ferritic steel material such as this one, 24 potential slip systems are considered for each grain, having $<111>$ slip directions, with $\{110\}$ or $\{112\}$ slip planes. All slip systems within a grain are assumed to have equal Critical Resolved Shear Stress (CRSS) at any state of deformation. The hardening behavior is defined at the microscopic level by prescribing the CRSS for any given grain as a function of the accumulated slip of that grain. The experimental stress-strain data along RD was used to calibrate the microscopic hardening law. A two-stage Voce-type law with 5 independent parameters was adopted of which the details can be found in Appendix 7.

\subsection{Results and discussion}

Since the VEF enables to simulate any arbitrary stress state, it is possible to directly assess its predictive accuracy by comparison with physical experiments. Figure 1 shows the experimentally acquired uniaxial stress-strain response along with the VEF simulation results. It can be inferred from Figures $1(\mathrm{~d})$ and $(\mathrm{g})$ that the VEF predictions for 45 degree and 90 degree tensile tests accurately reproduce the experimentally measured behaviour. Comparison with additional tensile experiments up to a nominal strain of 0.10 (see Figs.1(b),(c) and (f)) confirm the accuracy of the VEF in predicting the variation of plastic behavior with the direction.In addition, Figure 5 shows the comparison between experimentally measured $r$-values as a function of the tensile direction with respect to the $\mathrm{RD}$. The experimental values were measured using a gauge mark at an nominal strain of 0.1 . The measured values shown in Table 2 can be compared with those predicted by the VEF in Table 3. It can be seen from Fig. 5 that the VEF enables to predict the trend of the experimental $r$-value profile. The evolution of the $r$-values $r_{0}, r_{45}$ and $r_{90}$ as a function of the longitudinal plastic strain is shown in Fig.6. The $r$-values were calculated as a plastic strain ratio:

$$
r_{\alpha}=\frac{\epsilon_{w}^{p}}{-\epsilon_{l}^{p}-\epsilon_{w}^{p}}
$$

with $\epsilon_{w}^{p}$ and $\epsilon_{l}^{p}$ the plastic strain in the width and longitudinal direction, respectively. Both strain components were measured with DIC to calculate the evolution of the $r$-values according to Eq.(1). Likewise, the virtual data generated by the VEF are used to predict the $r$-value evolution. For the sake of completeness, the $r$-values manually measured using gauge marks (see table 2 ) are also shown. It can be inferred that the experimental $r$-values $r_{45}$ and $r_{90}$ tend to saturate as opposed to the VEF predictions which monotonically increase.

Fig. 3 shows that the VEF quite accurately reproduces the physical biaxial experiments. The onset of yielding under biaxial tension, however, is often not precisely captured by the VEF as this model does not account for gradual 
activation of slip systems within each grain, which occurs in reality upon beginning of plastic deformation. The concept of the contour of plastic work in stress space $[37,38]$ was adopted to assess the work hardening behavior under biaxial tension. After a certain amount of deformation such contours do not represent the actual yield loci. Indeed, the contours of plastic work are a collection of stress points associated with a specified amount of plastic work. The stress-strain curve obtained from a uniaxial tension test along the RD (Figure 1(a)) was used as a reference datum for work hardening. As such, this curve was used to determine the uniaxial true stress $\sigma_{0}$ and the plastic work per unit volume $W^{p}$ corresponding to particular values of the reference plastic strain $\epsilon_{0, \text { exp }}^{p}$. The uniaxial true stress $\sigma_{90}$ and the biaxial true stress components $\left(\sigma_{x}, \sigma_{y}\right)$ obtained from the biaxial tensile tests were then determined at the same plastic work $W^{p}$. The stress points $\left(\sigma_{0}, 0\right),\left(0, \sigma_{90}\right)$ and $\left(\sigma_{x}, \sigma_{y}\right)$ corresponding to a certain value of $\epsilon_{0}^{p}$ can be plotted in the principal stress space resulting in a contour of plastic work.

The contours of plastic work were determined for different levels of $\epsilon_{0, \exp }^{p}$ up to $\epsilon_{0, \text { exp }}^{p}=0.24$ as shown in Figure 7(a) along with the work contours predicted by the VEF. In general, the absolute stress levels of the predicted work contours are in good agreement with the measured work contours. The initial yielding under biaxial tension, however, is poorly predicted by the VEF. The latter can potentially be attributed to (i) the strong differential hardening $[37,38]$ during initial plastic deformation which cannot be captured by the ALAMEL model and (ii) the transition from elastic to plastic deformation which is not considered by the ALAMEL model.

The former (i) can be investigated in more detail by analysing the normalized work contours shown in Figure $7(\mathrm{~b})$. By normalizing the work contours shown in Figure $7(\mathrm{a})$ using $\sigma_{0}$ associated with a specific value of $\epsilon_{0 \text {,exp }}^{p}$, it can be appreciated that in the majority of the monitored stress states the contours of plastic work expand in the range $0<\epsilon_{0, \exp }^{p} \leq 0.03$. This particular phenomenon is referred to as differential hardening. It can be observed that the shape of the experimental work contours remain almost identical for $0.03<\epsilon_{0, \exp }^{p}<0.24$ meaning that isotropic hardening is valid in this particular strain range. Figure 7 (b) shows that the VEF captures a significantly weaker differential hardening than experimentally observed. The evolution of the shape of the work contours in a particular stress state can also be visualized by plotting the distance $l$ from the origin to the normalized stress point, as a function of the reference plastic strain:

$$
l=\sqrt{\left(\frac{\sigma_{x}}{\sigma_{0}}\right)^{2}+\left(\frac{\sigma_{y}}{\sigma_{0}}\right)^{2}+\left(\frac{\sigma_{x y}}{\sigma_{0}}\right)^{2}}
$$

where the stress components are defined with respect to the orthogonal material axes of which the x-axis is aligned with RD. As opposed to the biaxial tensile tests and the uniaxial tensile test in the RD and TD, the uniaxial tensile tensile tests oriented at an angle with respect to the RD involve a non-zero $\sigma_{x y}$. Figure 8 shows the evolution of the distance $l$ for biaxial tension (a) and uniaxial tension (b) as a function of the reference plastic strain $\epsilon_{0}^{p l}$ associated 
Table 3: Predicted $r$-values at an engineering strain $\epsilon_{\text {eng }}=0.10$ using the VEF.

\begin{tabular}{lll}
\hline$r_{0}$ & $r_{45}$ & $r_{90}$ \\
\hline 2.19 & 2.45 & 2.85
\end{tabular}

with consecutive work contours. It must be noted that Figures 8 (a) and (b) have a different scale. Nevertheless, it can be inferred that the general trend predicted by the VEF is in close agreement with the experimentally acquired data. Indeed, the VEF correctly predicts expansion $\left(\frac{d l}{d \epsilon_{0}^{p}}>0\right)$ and shrinkage $\left(\frac{d l}{d \epsilon_{0}^{p}}<0\right)$ of the work contours, except for the stress ratio $\sigma_{x}: \sigma_{x}=1: 2$. The strong change of $l$ during the initial deformation, however, cannot be captured by the VEF. A similar observation for another steel grade is reported in [35]. Finally, figure 9 compares the experimentally measured direction of plastic strain rates $\beta$ with the predicted ones by VEF as a function of the reference plastic strain $\epsilon_{0}^{p}$. Figure $9(\mathrm{a})$ shows the direction of plastic strain rate $\beta$ for the biaxial stress paths where $\beta$ is defined as (see Figure 13(d) for a schematic representation):

$$
\beta=\tan ^{-1} \frac{\dot{\epsilon}_{y}}{\dot{\epsilon}_{x}}
$$

Figure $9(\mathrm{~b})$ shows $\beta$ for the uniaxial tensile testing under various angles with the $\mathrm{RD}$ where $\beta$ is defined as:

$$
\beta=\tan ^{-1} \frac{\dot{\epsilon}_{w}}{\dot{\epsilon}_{l}}
$$

with $\dot{\epsilon}_{w}$ and $\dot{\epsilon}_{l}$ the width and longitudinal strain rate, respectively. Eq.(3) and Eq.(4), however, are equivalent for a uniaxial tensile test in the RD. It can be inferred from Figure 9 that the $\beta$-values are almost constant and accurately predicted by the VEF. 


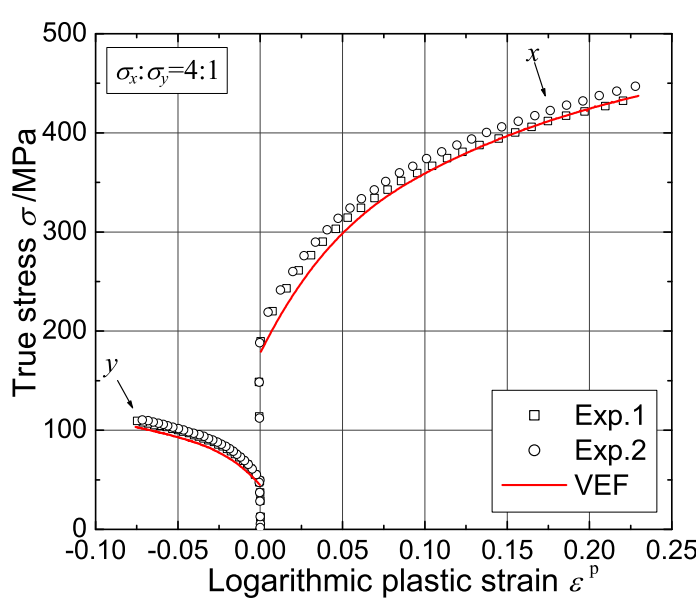

(a)

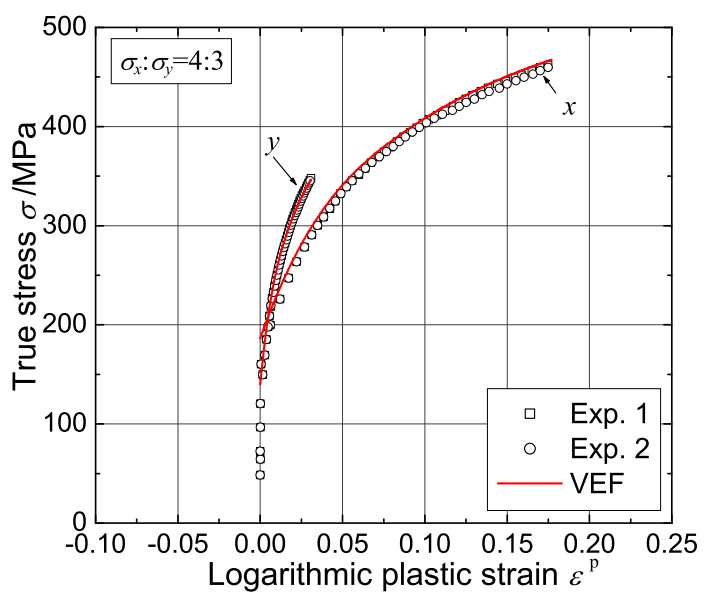

(c)

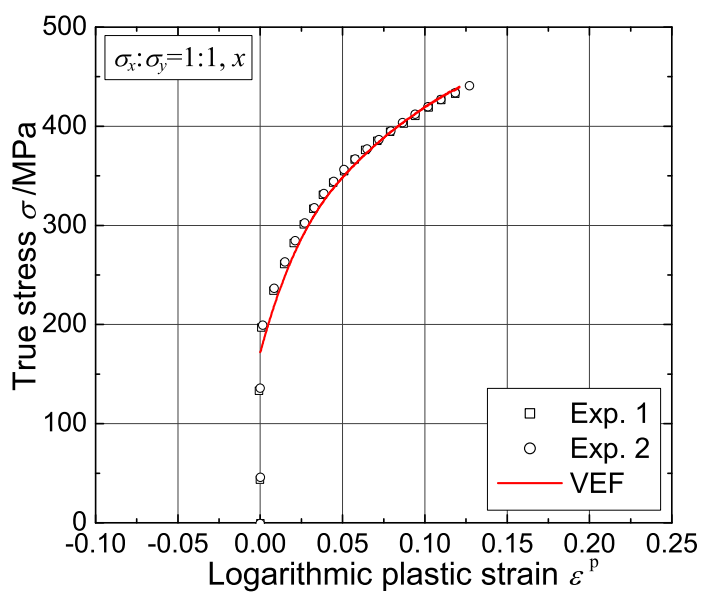

(e)

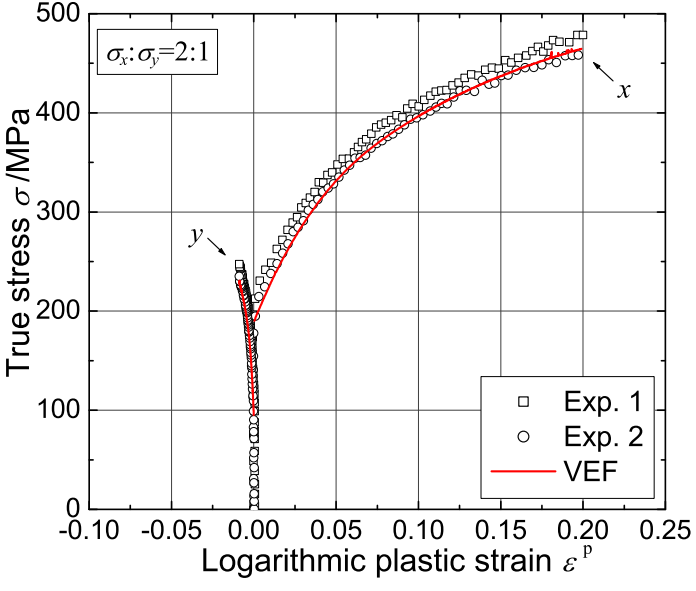

(b)

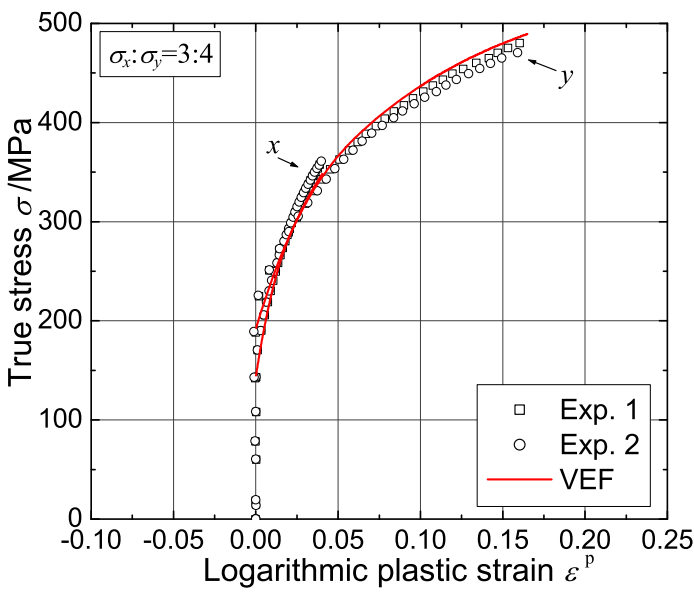

(d)

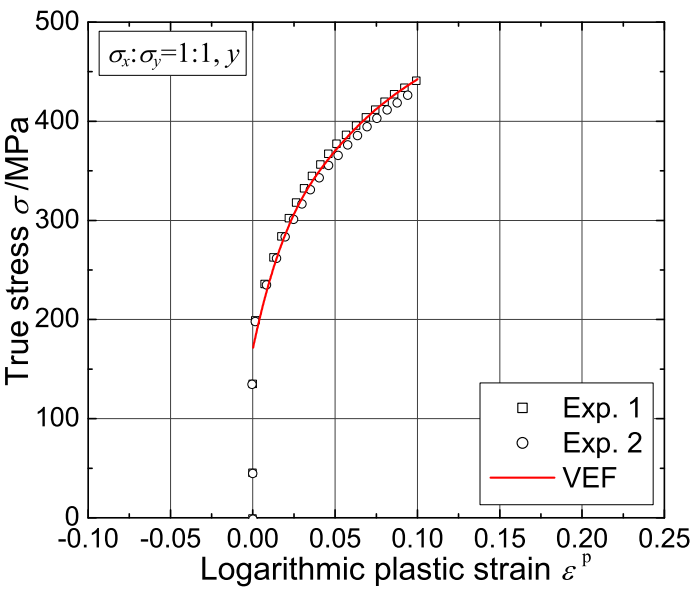

(f)

Fig. 3: Biaxial stress-strain curves.(a) $\sigma_{x}: \sigma_{y}=4: 1$ (b) 2:1 (c) 4:3 (d)3:4 (e) $1: 1, \mathrm{x}$ and (f) $1: 1, \mathrm{y}$ 


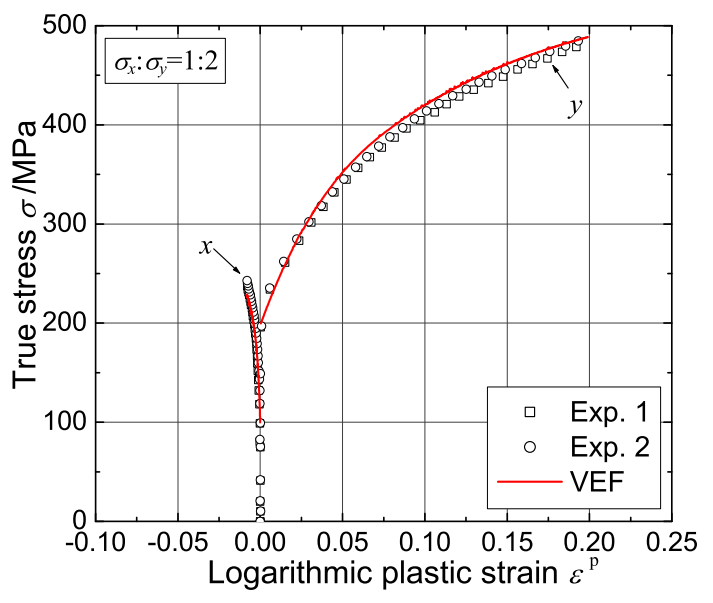

$(\mathrm{g})$

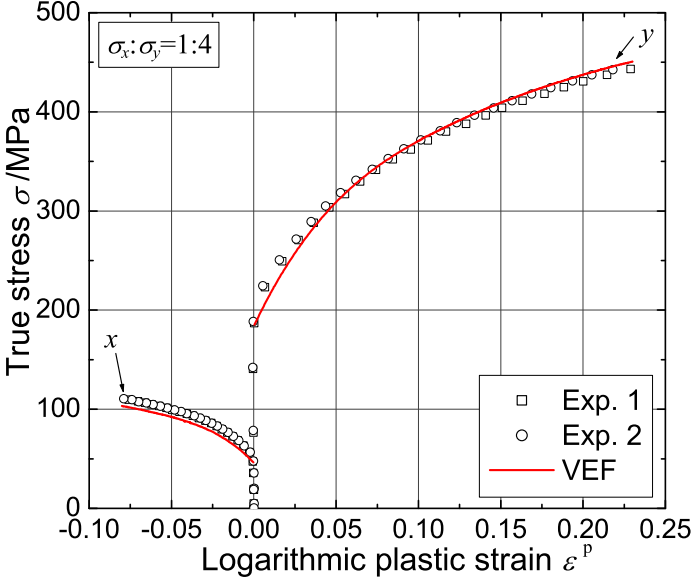

(h)

Fig. 3: (Cont'd) Biaxial stress-strain curves.(g) $\sigma_{x}: \sigma_{y}=1: 2$ (h) 1:4

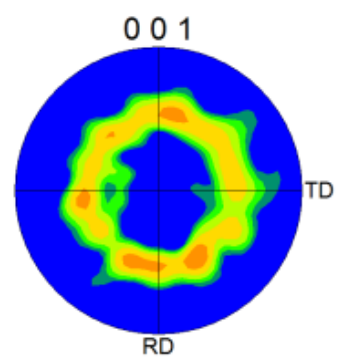

111

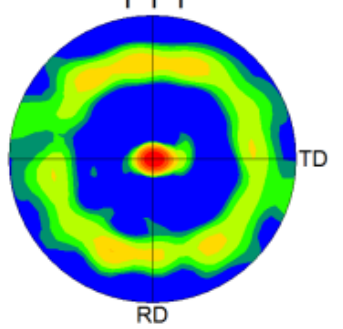

(a)
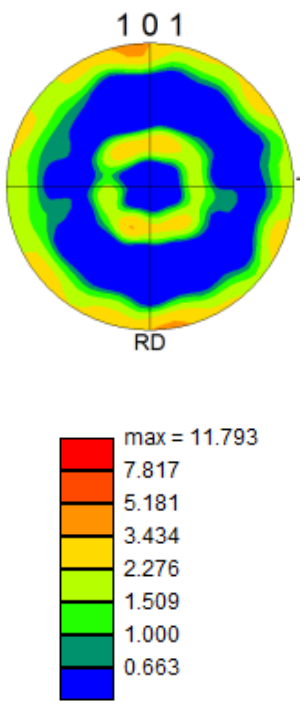

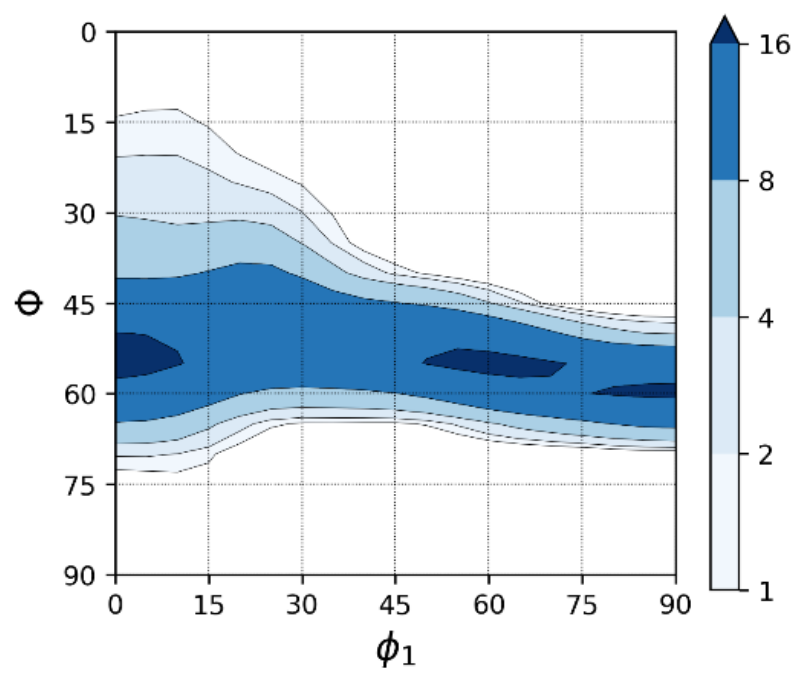

(b)

Fig. 4: (a) The (001), (101) and (111) pole figures at sheet mid-thickness.(b) The $\phi_{2}=45^{\circ}$-section of the Orientation Distribution Function (average across sheet thickness). 


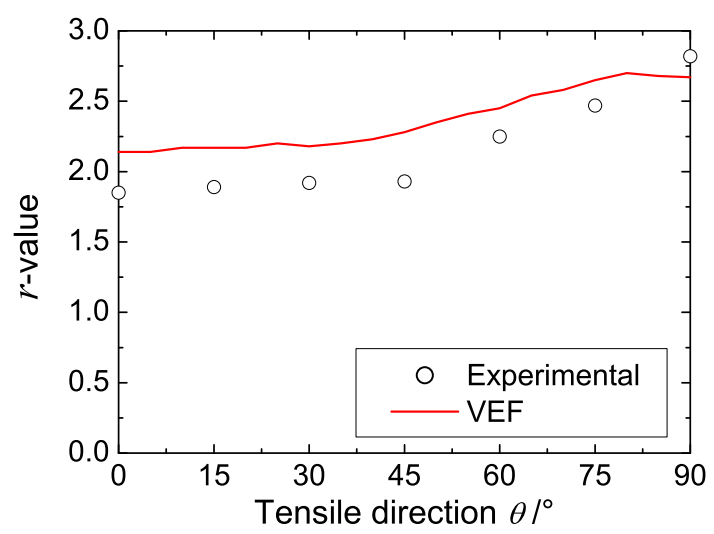

Fig. 5: Experimental and predicted $r$-value (at engineering strain $\epsilon_{e n g}=0.10$ )as a function of the tensile direction. 


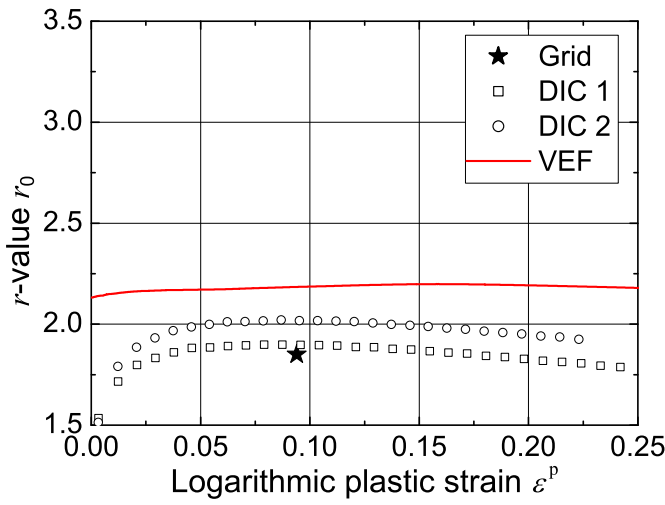

(a)

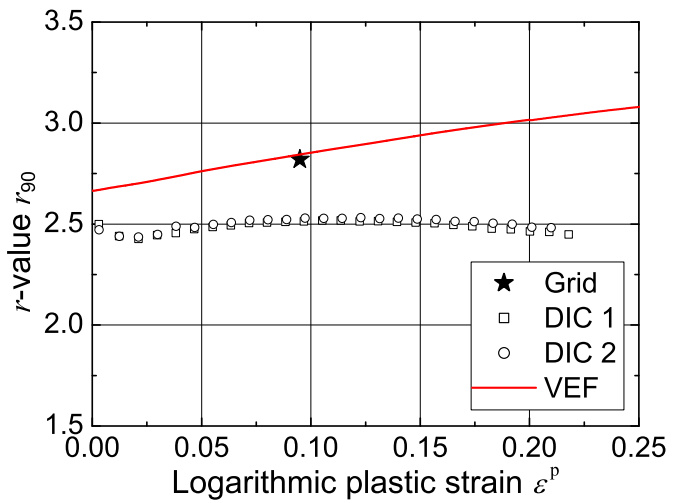

(c)

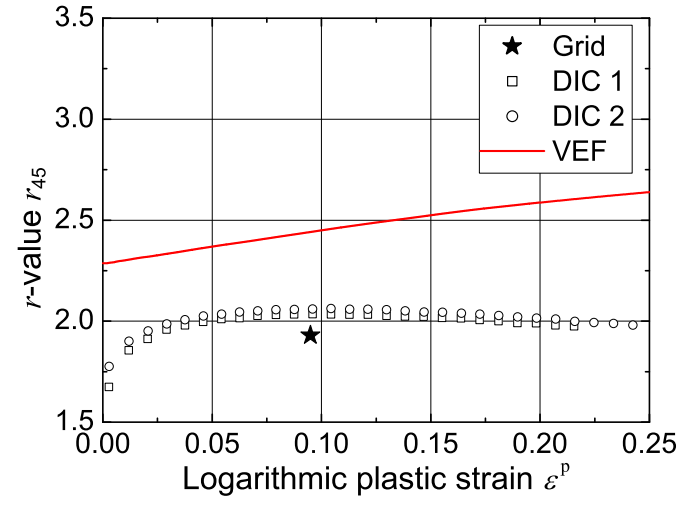

(b)

Fig. 6: Evolution of the $r$-value as a function of longitudinal plastic strain.(a) $r_{0}$ (b) $r_{45}$ and (c) $r_{90}$. 


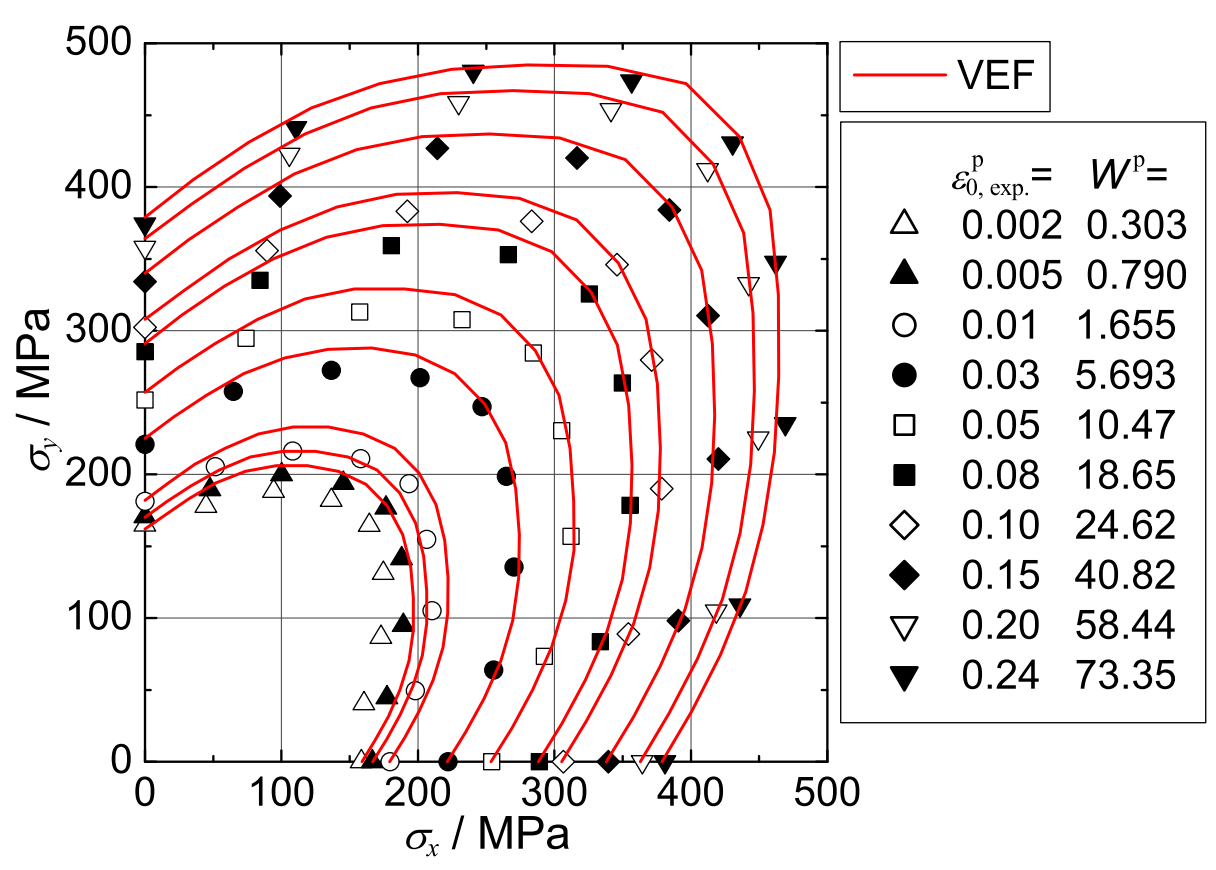

(a)

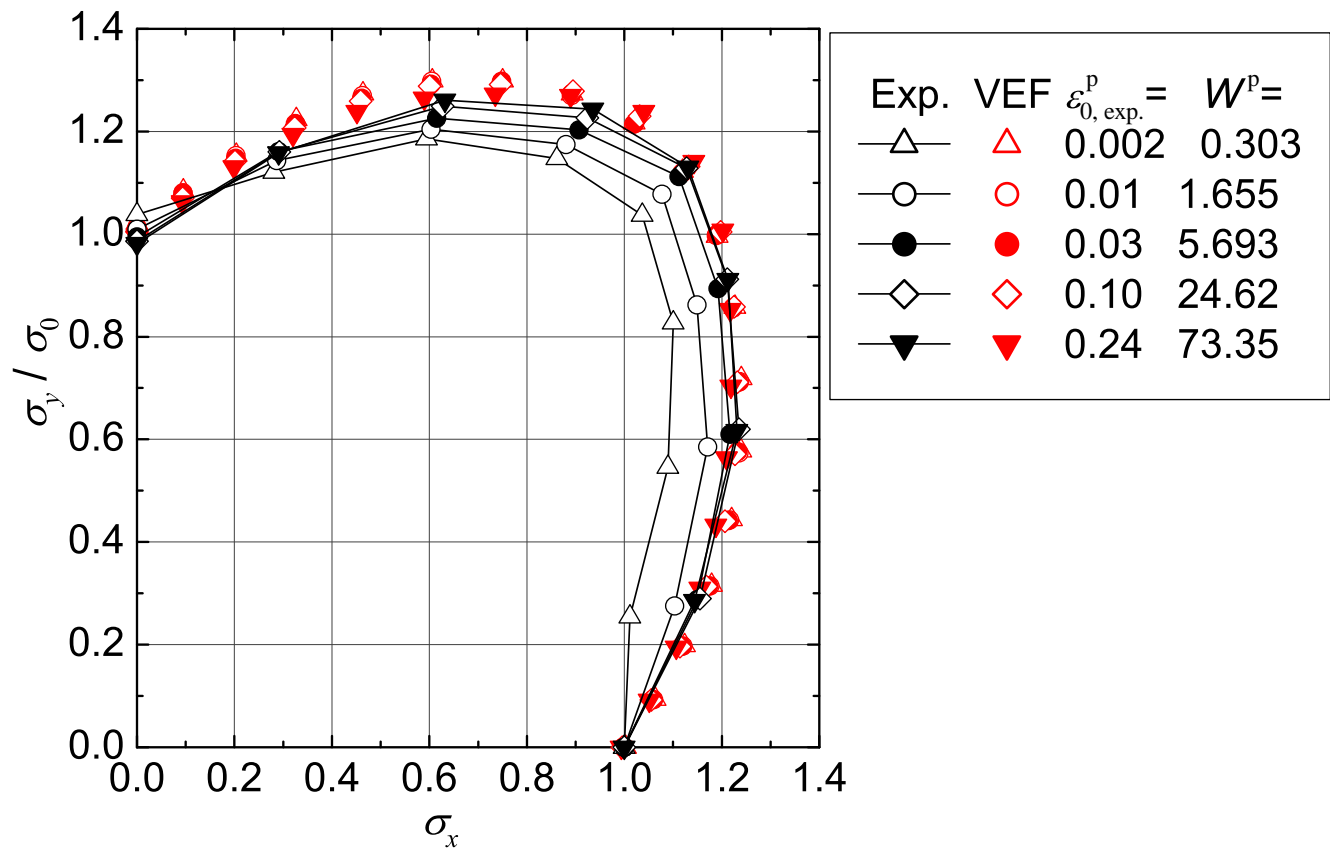

(b)

Fig. 7: (a) Experimental and predicted work contours (b) Experimental and predicted work contours normalized by $\sigma_{0}$. 


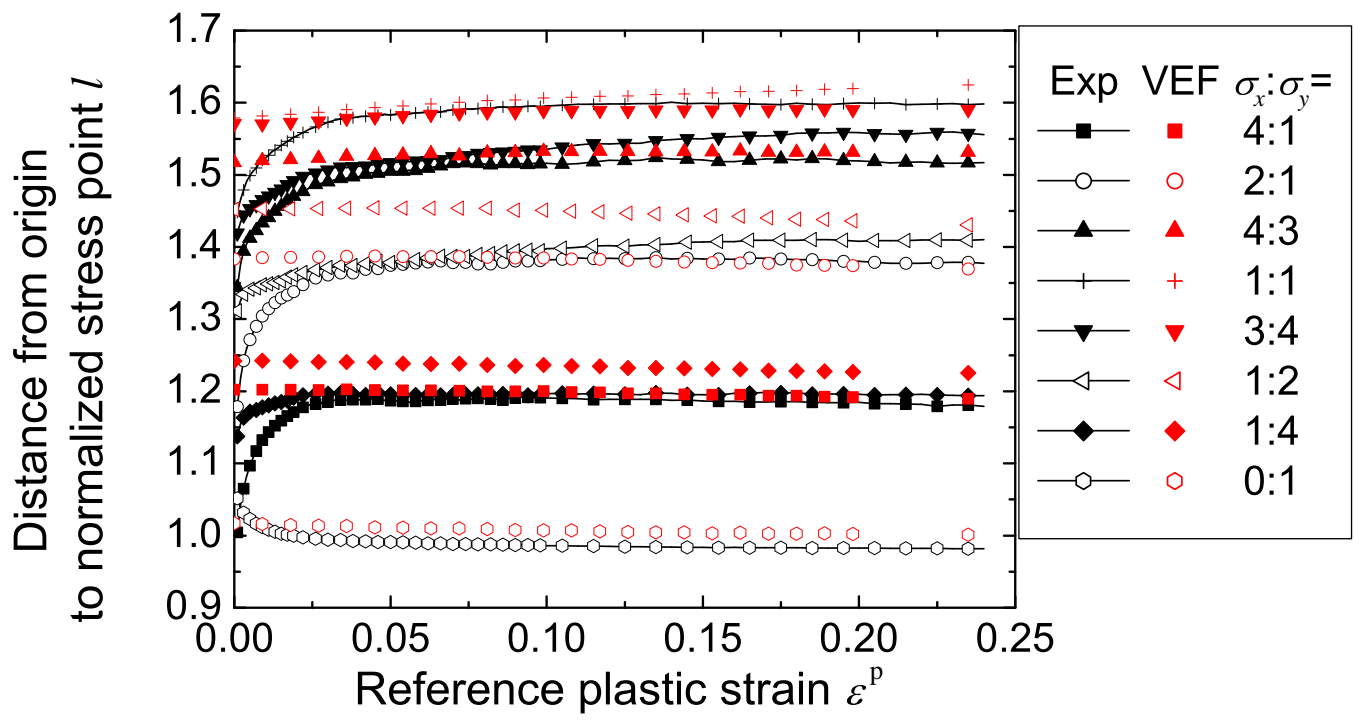

(a)

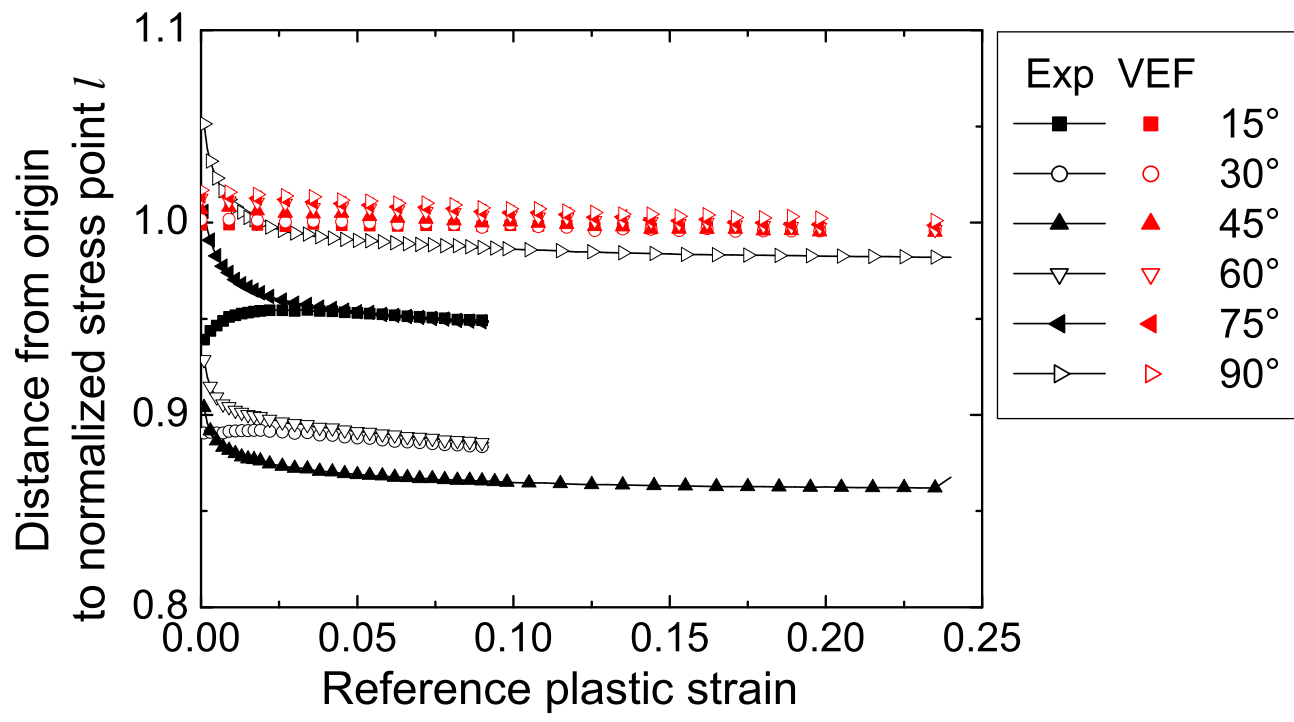

(b)

Fig. 8: Evolution of the distance $l$ from origin to normalized stress point as a function of the reference plastic strain for different stress states. a) Biaxial tension b) Uniaxial tension 


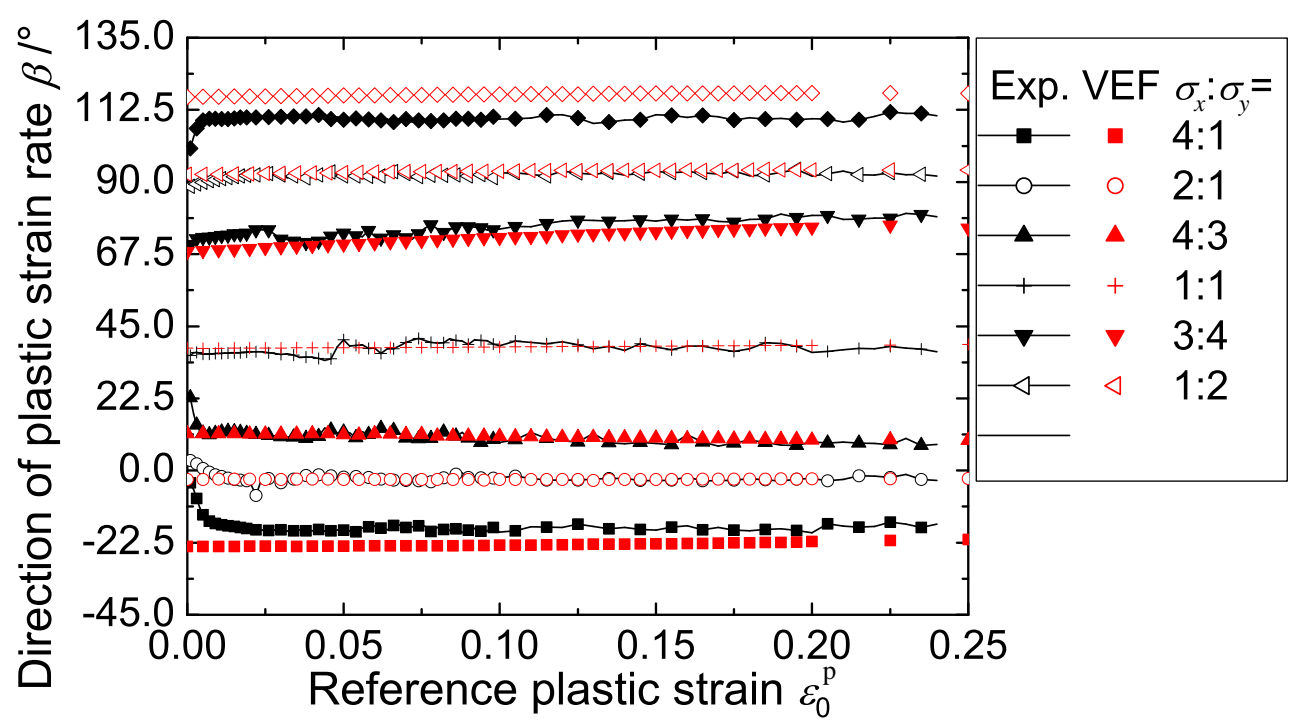

(a)

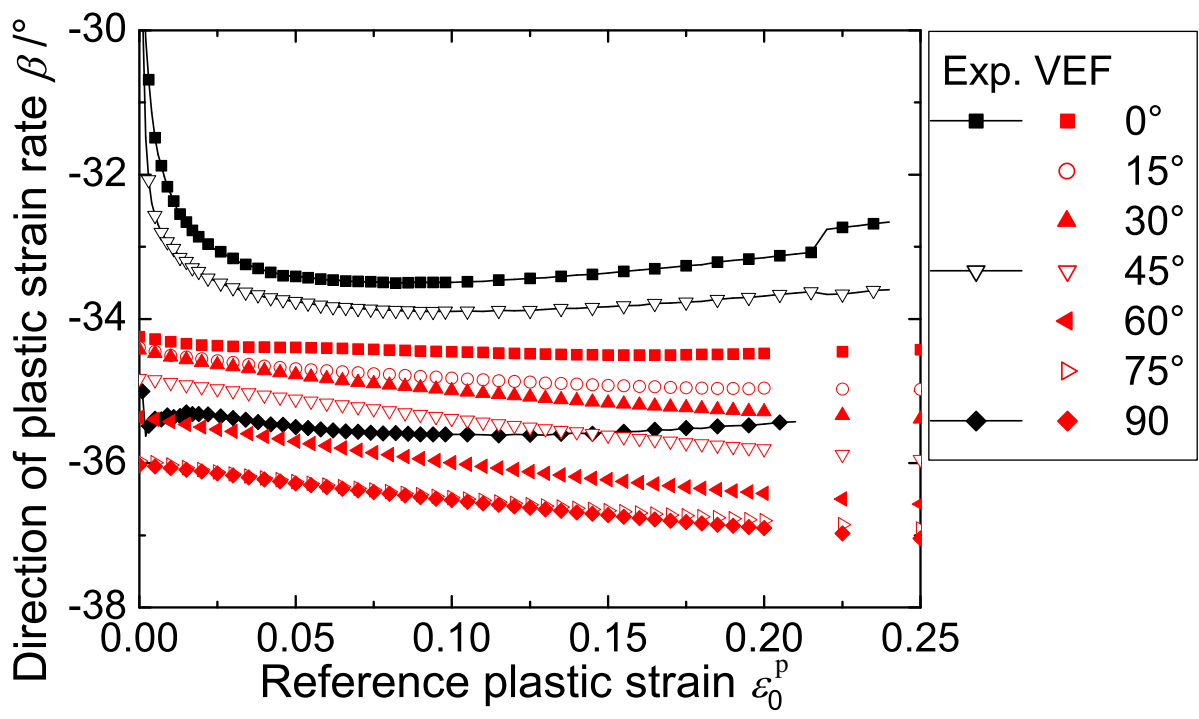

(b)

Fig. 9: Direction of plastic strain rate as a function of the reference plastic strain for different stress states. a) Biaxial tension b) Uniaxial tension 
3 Calibration of anisotropic yield functions

In this section three selected plane stress yield functions (the von Mises criterion [39], Hill's 48 [40] and the Yld2000-2d [41,42] yield loci) are calibrated using physical and virtual test data. An overview of the parameters required to calibrate the selected yield criteria can be found in table 4. Swift's strain hardening law was used to describe the reference strain hardening curve measured through a tensile test in the RD of the test material, see Figure 1(a) and the parameters in Table 2. The latter curve completely determines the von Mises yield criterion. Figure 11 (a) shows a selected number of work contours along with the calibrated von Mises yield criterion. It can be inferred that initial yielding can be accurately described by the von Mises yield locus. At a reference plastic strain of about 3\%, however, the von Mises yield criterion fails to precisely describe the plastic material response in the vicinity of quasi-balanced biaxial tension. The plane stress version of Hill's 48 yield criterion was calibrated by using the $r$-values and the reference strain hardening curve. The experimental (Table 2) and virtual (Table 3) $r$-values were used to calibrate Hill's 48 criterion. The calibrated yield loci are shown in Fig. 11(b). The r-based version of the Hill's yield criterion obviously cannot accurately describe yielding in the first quadrant of stress space. However, it must be noted that the yield loci calibrated using experimental (Exp) and virtual (VEF) $r$-values are very similar as can be seen from Fig. 11(b).

The material parameters $\alpha_{i}(\mathrm{i}=1-8)$ of the Yld2000-2d yield function were calibrated using the parameters indicated in Table 5 while the exponent $M$ was identified by minimizing the root mean square error $\delta_{l}$ between the normalized work contour and the Yld2000-2d yield locus:

$$
\delta_{l}=\sqrt{\frac{\sum_{i}^{N}\left(l_{i}^{\prime}\left(\varphi_{i}\right)^{2}-l_{i}\left(\varphi_{i}\right)^{2}\right)}{N}}
$$

with $\varphi_{i}$ the loading angle of the $i$-th stress point from the $x$-axis in the principal stress space, $l_{i}$ the distance between the origin of the principal stress space and the i-th stress point, $l_{i}^{\prime}$ the distance between the origin of the principal stress space and the theoretical yield locus along the loading direction $\varphi_{i}$ and $N(=9)$ the total number of stress paths. The latter is schematically represented in Figure 10.

Calibration can be done for several levels of reference plastic strain $\epsilon_{0}^{p}$ to account for differential hardening as proposed by Kuwabara et al.[1]. It can be inferred from Fig.8, however, that the shape of the yield locus remains constant as from $\epsilon_{0}^{p}=3 \%$. Beyond that value, the plastic work contours are homothetic and isotropic hardening is valid. Consequently, the yield function can be calibrated at an arbitrary reference plastic strain in the range $0.03<$ $\epsilon_{0}^{p} \leq 0.24$. It has been shown that for such a constitutive material behavior the effect of considering differential hardening in a hydraulic bulge test simulation can be safely ignored [43].

In this study, the Yld2000-2d yield function is calibrated at a reference plastic strain of $\epsilon_{0}^{p}=0.24$ using experimental (Exp) and virtual (VEF) data shown 


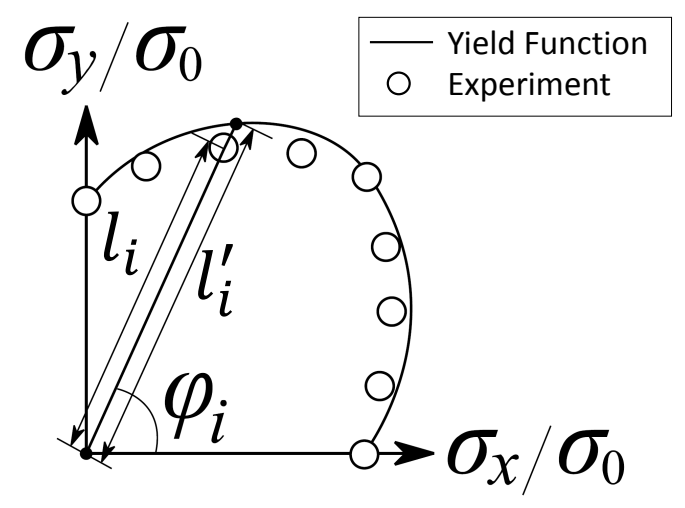

Fig. 10: Schematic illustration for calculating $\delta_{l}$.

in Figure 7. The resulting yield loci and the identified anisotropic parameters can be found in Fig.11(c) and Table 5, respectively.

It can be observed that both calibrated yield loci correspond accurately with the experimental data beyond a reference plastic strain of $3 \%$. More important in the context of this paper is the observation that both calibrations lead to almost identical yield loci.

The calibrated yield functions can be used to theoretically predict the $r$ value profile and the results are shown in Figure 12. The von Mises criterion cannot reproduce the directional variation of the plastic behavior while Hill's 48 criterion and the Yld2000-2d function yield very similar predictions. The best agreement is found when experimental data is used to calibrate the selected anisotropic yield functions, which is reasonable considering that the experimental $r$-values were used in the calibration with experimental data but not in the calibration with virtual data (VEF).

Finally, Figure 13 compares the direction of plastic strain rates $\beta$ measured at different levels of $\epsilon_{0}^{p}$ with those calculated by the selected yield functions. It can be seen that even the isotropic von Mises criterion quite accurately predicts the direction of the plastic strain rate. Hill's 48 criterion seems to be less accurate in predicting the direction of plastic strain rate. It can be inferred that Yld2000-2d outperforms all other yield criteria in predicting $\beta$ and that the results are independent of the calibration data (experimental or virtual). 
Table 4: Material parameters used to calibrate the selected yield functions.

\begin{tabular}{cccc}
\hline Material Parameter & von Mises & Hill's 48 & Yld2000-2d \\
\hline & & & \\
$\sigma_{0}$ & $\checkmark$ & $\checkmark$ & $\checkmark$ \\
$\sigma_{45}$ & & & $\checkmark$ \\
$\sigma_{90}$ & & & $\checkmark$ \\
$\sigma_{b}$ & & $\checkmark$ & $\checkmark$ \\
$r_{0}$ & & $\checkmark$ & $\checkmark$ \\
$r_{45}$ & & $\checkmark$ & $\checkmark$ \\
$r_{90}$ & & & $\checkmark$ \\
$r_{b}$ & & & $\checkmark$ \\
\hline
\end{tabular}

Table 5: Calibrated Yld2000-2d parameters at a reference plastic strain $\epsilon_{0}^{p}=0.24$.

\begin{tabular}{cccccccccc}
\hline & $\alpha_{1}$ & $\alpha_{2}$ & $\alpha_{3}$ & $\alpha_{4}$ & $\alpha_{5}$ & $\alpha_{6}$ & $\alpha_{7}$ & $\alpha_{8}$ & $\mathrm{M}$ \\
\hline Calibration Data & & & & & & & & & \\
Exp & 0.9394 & 1.1841 & 0.8872 & 0.8765 & 0.9333 & 0.8020 & 1.0462 & 1.0227 & 5.90 \\
VEF & 0.9950 & 1.1232 & 0.8785 & 0.8672 & 0.9119 & 0.7867 & 1.0678 & 0.9599 & 5.83 \\
\hline
\end{tabular}




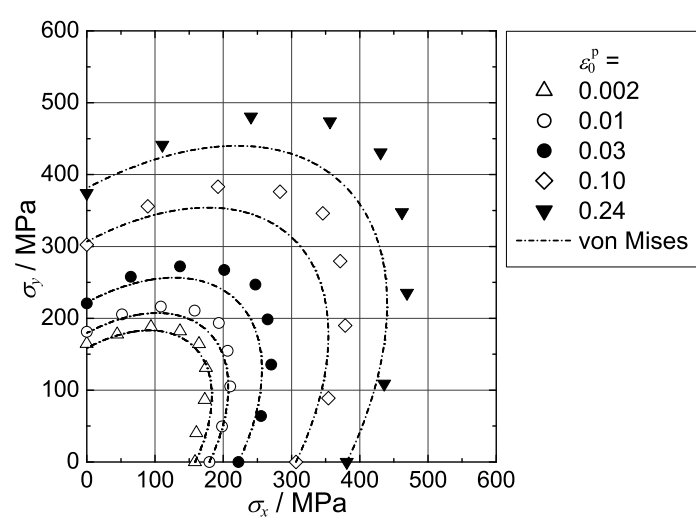

(a)

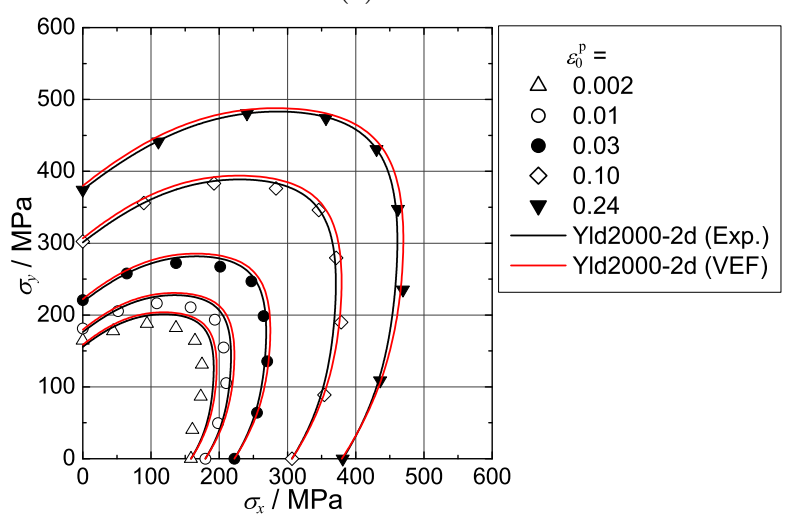

(c)

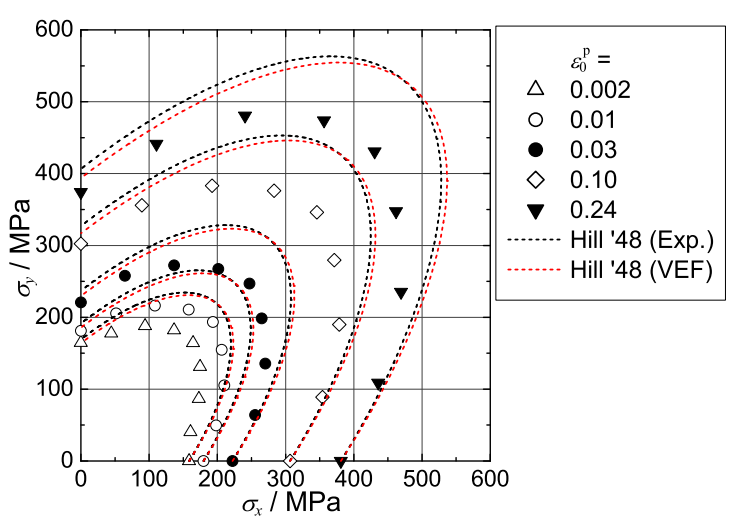

(b)

Fig. 11: Calibrated yield functions and experimental work contours at selected values of $\epsilon_{0}^{p}$. (a) von Mises (b) Hill'48 (c) Yld2000-2d 


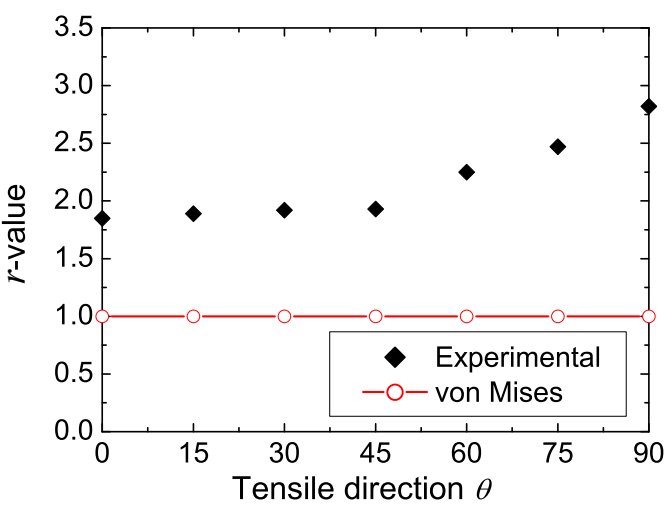

(a)

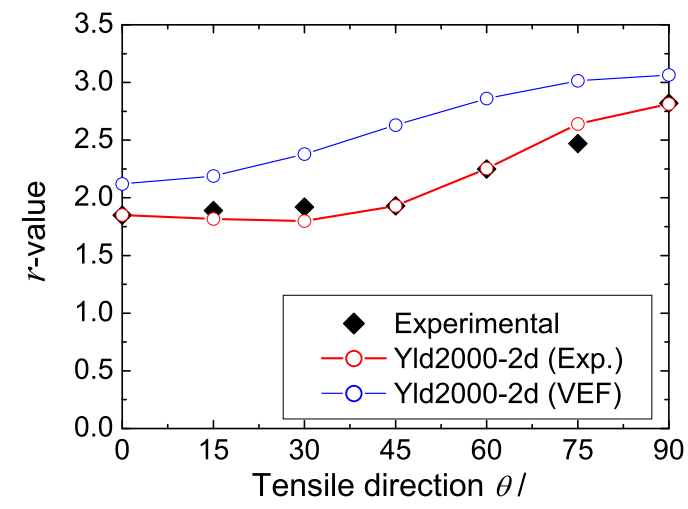

(c)

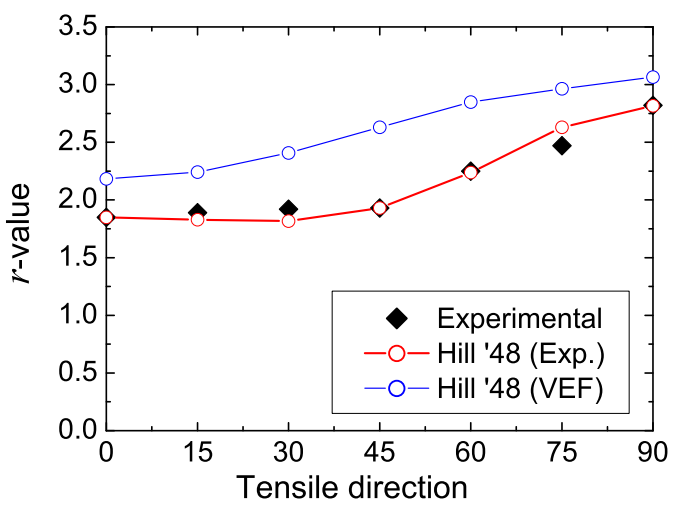

(b)

Fig. 12: Theoretical prediction of the $r$-value as a function of the tensile direction in the RD. (a) von Mises (b) Hill'48 (c) Yld2000-2d 


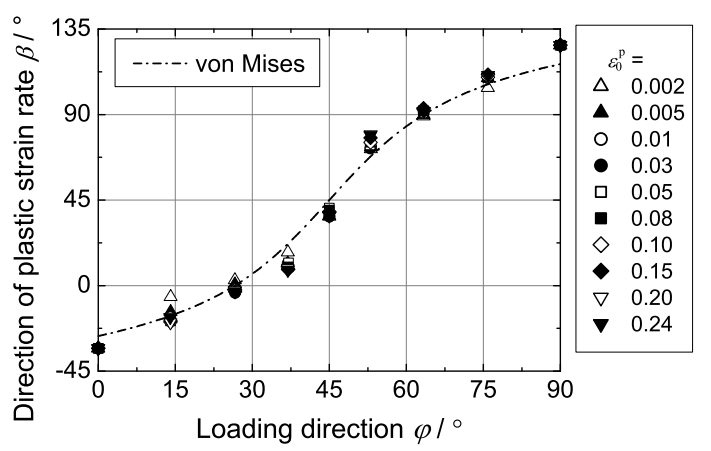

(a)

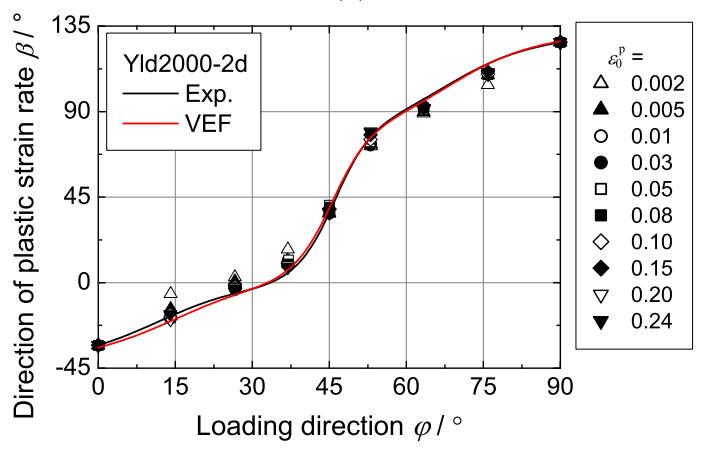

(c)

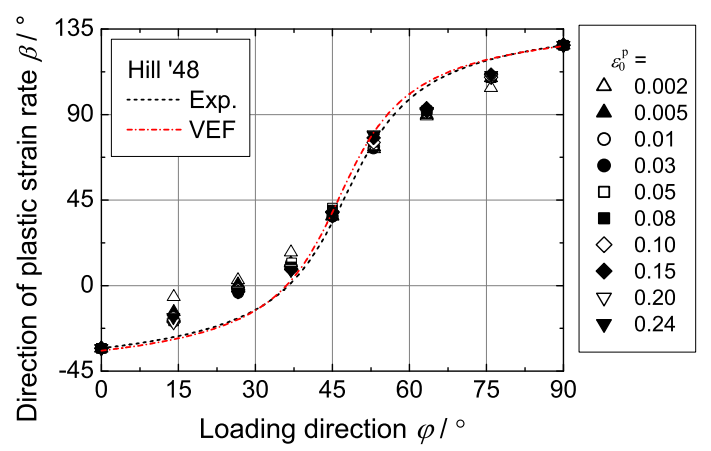

(b)

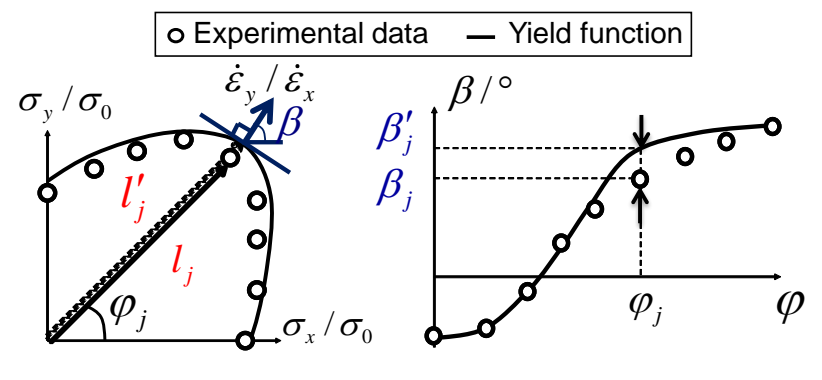

(d)

Fig. 13: Theoretical prediction of $\beta$ as a function of the loading angle $\varphi$.(a) von Mises (b) Hill'48 (c) Yld2000-2d and (d) Schematic illustration for calculating $\beta$ as a function of $\varphi$. 


\section{Hydraulic bulge test}

In section 2 it was shown that, for the test material under investigation, plasticity experiments in the first quadrant of stress space can be accurately reproduced by the VEF. In section 3, the virtual test data was successfully used to calibrate the selected yield functions. In this section, hydraulic bulge tests and FE simulations are performed to evaluate the application of the calibrated yield functions.

\subsection{Experimental}

The hydraulic bulge test consists of clamping a circular blank and exposing it to a hydraulic pressure. The stress state generated in the bulged specimen ranges from quasi-balanced biaxial tension at the apex to plane strain close to the clamping region. As such, the bulge test enables to probe the test material in a portion of the first quadrant of stress space. Figure 14 shows the experimental set up and a schematic drawing of the forming tools used for hydraulic bulge forming. The diameter of the blank holder opening was 150 $\mathrm{mm}$ and the die profile radius was $8 \mathrm{~mm}$. The material flow-in was prevented by a triangular draw bead. No lubricant was used at the interface between the blank and die surface. The hydraulic cylinder was coupled with a Zwick elektromechanical servo testing actuator enabling to control the flow rate of the hydraulic fluid forming the bulge. The latter is required to keep the thickness strain rate $\dot{\epsilon}_{z}$ at the apex constant [44]. In this study, the velocity of the hydraulic cylinder was controlled in such a way that the equivalent plastic strain rate was kept constant to approximately $10^{-4} \mathrm{~s}^{-1}$. The latter value is consistent with the material tests presented in section 2 . The required velocity profile was investigated by conducting a reference experiment with a constant flow rate. This result was linearly scaled to obtain the velocity profile. The thickness strain $\left|\epsilon_{z}\right|$ at the apex of the bulged specimen was measured using stereo DIC assuming volume constancy and ignoring elastic deformation:

$$
\left|\epsilon_{z}\right|=\left|-\epsilon_{1}-\epsilon_{2}\right|
$$

with $\epsilon_{1}$ and $\epsilon_{2}$ the principal strains. Figure 15 shows the instantaneous strain rate $\frac{\left|d \epsilon_{z}\right|}{d t}$ at the apex during bulge testing up to maximum uniform strain. It can be inferred that the adopted velocity profile enables to keep the strain rate close to $10^{-4} s^{-1}$. The pressure was measured by converting the load cell reading mounted on the Zwick actuator. The load cell reading was synchronized with the DIC system taking pictures at $1 \mathrm{~Hz}$. The optimal DIC settings can be found in table 6. Two Manta (Allied Vision) cameras were used along with Kowa lenses with a focal length of $\mathrm{f}=25 \mathrm{~mm}$. MatchID 3D [45] was used to post-process the images. 


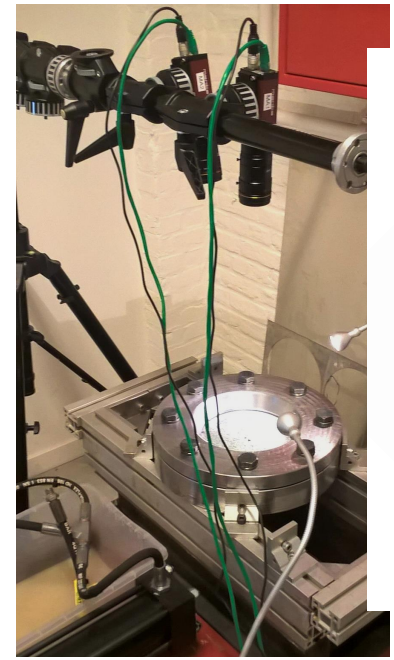

(a)

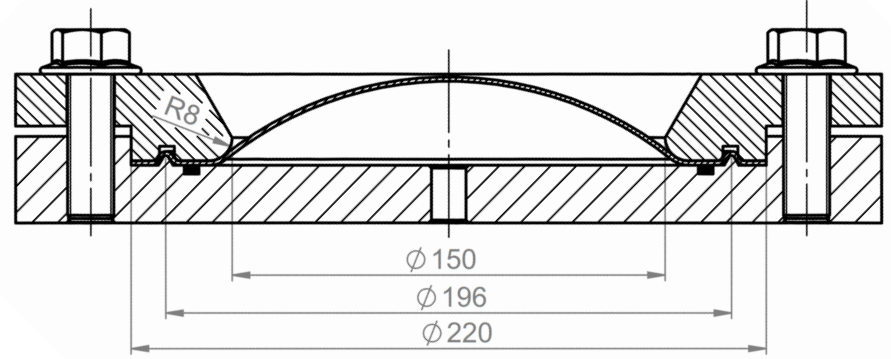

(b)

Fig. 14: (a)Experimental set up of the hydraulic bulge test (b) and schematic drawing.

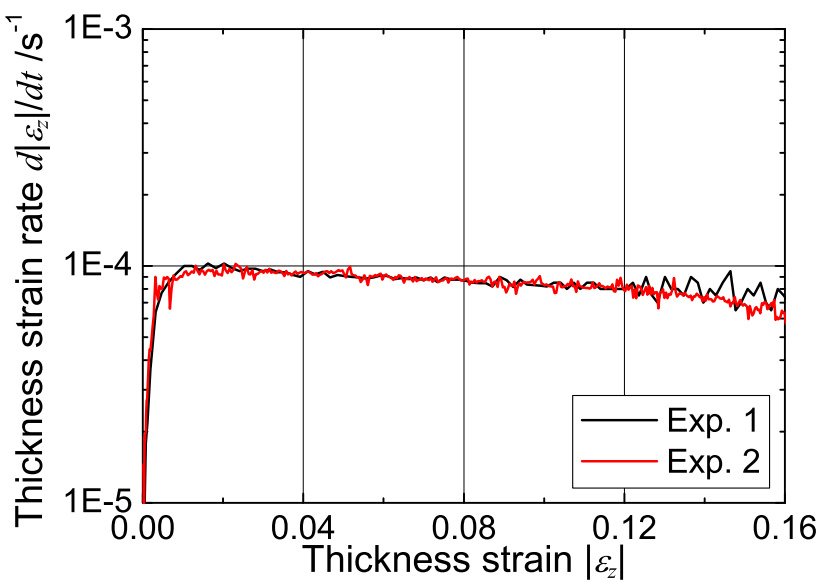

Fig. 15: Thickness strain rate at the apex as a function of the thickness strain.

\subsection{Numerical}

Abaqus/Standard [46] was used to simulate the hydraulic bulge test. Figure 16 shows the 3D FE model of which only one quarter was modelled given (i) the axial symmetry of the process and (ii) the orthotropic plastic anisotropy of the material. The FE model contains quadrilateral shell elements with reduced integration (S4R). The draw bead in this model was simplified by assuming 
Table 6: DIC parameters in the bulge test

\begin{tabular}{ll}
\hline DIC implementation & Specification \\
\hline Matching criteria & ZNSSD \\
Interpolation & Bicubic Interpolation \\
Transformation & Affine \\
Subset & 21 pixels \\
Step & 10 pixels \\
Spatial resolution & 21 pixels $=1.74 \mathrm{~mm}$ \\
In-plane displacement resolution & $5.49 \cdot 10^{-4} \mathrm{~mm}$ \\
Out-of-plane displacement resolution & $4.32 \cdot 10^{-3} \mathrm{~mm}$ \\
\hline Strain Smoothing & Quadratic Quadrilateral $(\mathrm{Q} 8)$ \\
Strain window size & 15 Datum points \\
Strain resolution & $7.26 \cdot 10^{-5}$ \\
\hline
\end{tabular}

that the nodal displacements at the draw bead position are zero. The blank holder force required for forming the draw bead was ignored in this model while a Coulomb friction coefficient of 0.3 is used between the sheet and the blank holder. Uniform pressure was applied in the FE model to represent the hydraulic fluid. The validation of the assumptions with respect to the boundary conditions (i.e. the draw bead and the blank holder force) were investigated with an axisymmetric FE model shown in Fig.16(b). The latter model takes the draw bead mechanics prior to bulging into account. An axisymmetric FE model, however, is confined to an isotropic material model. As such, both FE models shown in Fig.16 assuming a von Mises material are used to scrutinize the effect of simplifying the boundary conditions. The axisymmetric FE model contained 12 solid elements (CAX4R) through the thickness of the sheet.

The thickness strain at the apex as function of the pressure of the hydraulic fluid $\left(\left|\epsilon_{z}\right|-P\right)$ curves predicted by both FE models can be found in figure 17. It can be inferred that the difference between the 3D shell model (3D) and the axisymmetric FE model (2D) is marginal and within the experimental scatter of the experiments shown in Fig.18. The 2D simulation reveals that the blank is slightly pre-strained after forming the draw bead with a blank holder force of $F_{B H}=300 \mathrm{kN}$. However, it can be concluded that the draw bead mechanics can be safely ignored in the 3D FE model. Both FE models, however, assumed a friction coefficient of 0.3 . To scrutinize the effect of the frictional condition, an additional 3D simulation was performed assuming a friction coefficient of 0.1. The result (referred to as $3 D+$ friction 0.1 ) is shown in Figure 17 and it can be concluded that the frictional condition is of minor importance in the hydraulic bulge test.

This basically means that the $\left|\epsilon_{z}\right|-P$ curve is independent of the boundary conditions and the frictional behavior during bulging. Moreover, it has been verified that the $\left|\epsilon_{z}\right|-P$ curve is insensitive to the adopted mesh provided that a mesh convergence study is performed. In other words, the $\left|\epsilon_{z}\right|-P$ curve lends itself to assess the accuracy of the continuum plasticity model adopted in the FE model. Indeed, the evaluation of the plasticity model in the first quadrant 


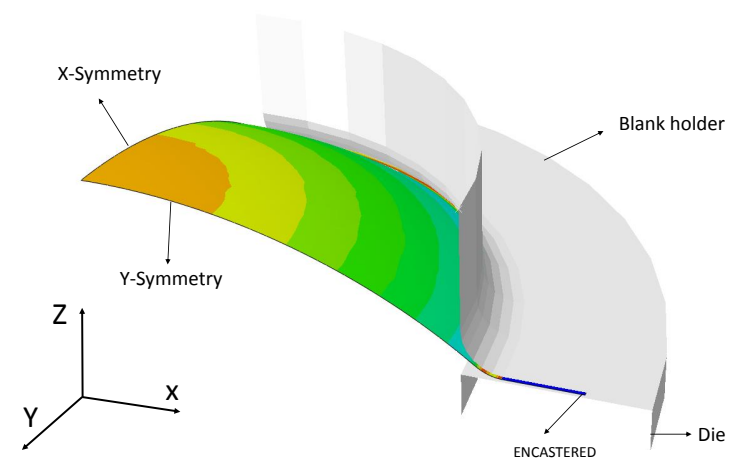

(a)

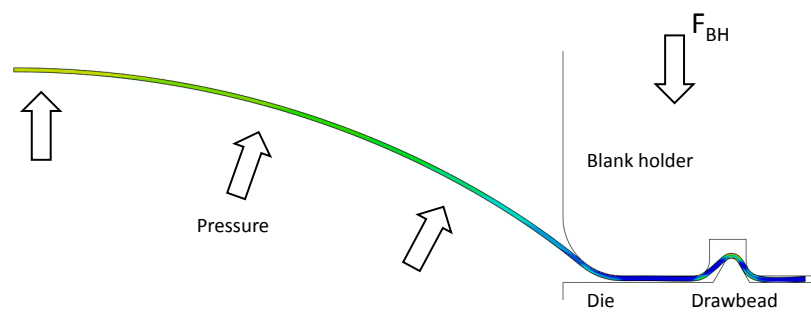

(b)

Fig. 16: Numerical modelling hydraulic bulge test. (a) 3D FE model (b) 2D FE model.

of stress space is not hampered by uncertainties with respect to frictional conditions, boundary conditions and technological aspects of the FE model.

\subsection{Results and discussion}

Figure 18 shows the experimentally acquired $\left|\epsilon_{z}\right|-P$ curve along with the simulations using the strain hardening behavior and anisotropic yield functions calibrated in section 2.1 and 3, respectively. It can be inferred that the von Mises criterion largely underestimates the pressure to form the bulge while Hill's 48 criterion overestimates the pressure. The Yld2000-2d yield function shows the best agreement with the experiment. More important in view of the goal of this paper, is that the virtually calibrated yield functions (referred to as VEF) yield an equivalent simulation accuracy as those calibrated using experimental data. The calibration procedure adopted for the Yld2000-2d functions yields almost identical results. Finally, Fig. 19 shows the measured thickness strain along the meridian line (RD) for a bulged specimen formed with a pressure of 


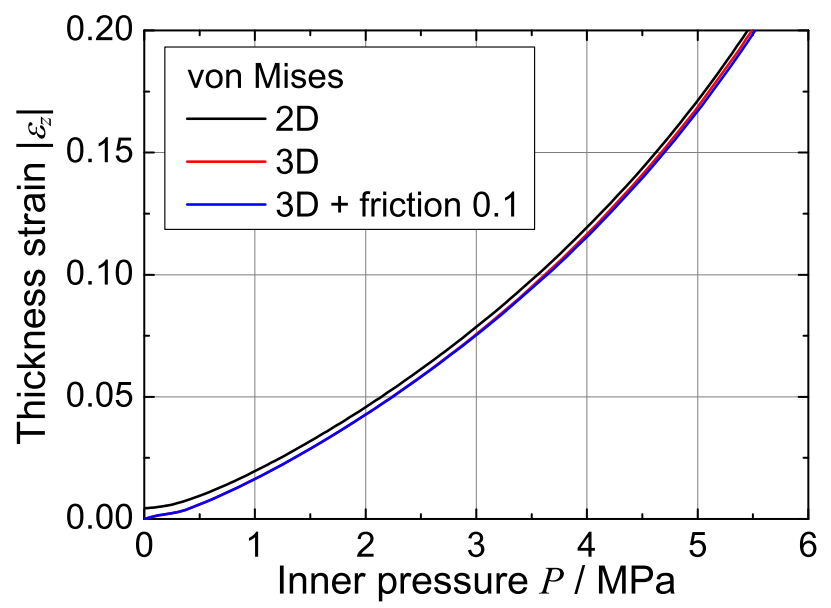

Fig. 17: Validation of simplified boundary conditions in a hydraulic bulge test simulation.

$P=6.4 \mathrm{MPa}$. The bulged specimen was carefully sectioned and the thickness along the meridian line was measured using a digital measurement gauge with a resolution of $0.001 \mathrm{~mm}$. As opposed to the von Mises criterion, the Hill's 48 yield criterion underestimates the strain levels. The Yld2000-2d yield function most accurately captures the strain distribution. The experimentally and virtually calibrated Yld2000-2d yield functions are in very close agreement. The thickness reduction at the apex is slightly underestimated while the difference reduces when approaching a radial distance of $70 \mathrm{~mm}$. The latter can be qualitatively explained by analysing the shape of the work contours, see Fig.11(c). It can be observed that the calibrated Yld2000-2d function is inaccurate at initial yielding under biaxial tension. The difference in the first $3 \%$ of reference plastic strain is significant in the vicinity of quasi-balanced biaxial tension. Indeed, in this region the flow stress is overestimated which could potentially explain the underestimation of the thickness reduction at the apex. However, Figure 11 (c) shows that the difference under plane strain conditions between the experimental work contour and Yld2000-2d rapidly vanishes, hence the accurate prediction of the strain distribution close to the clamping region. 


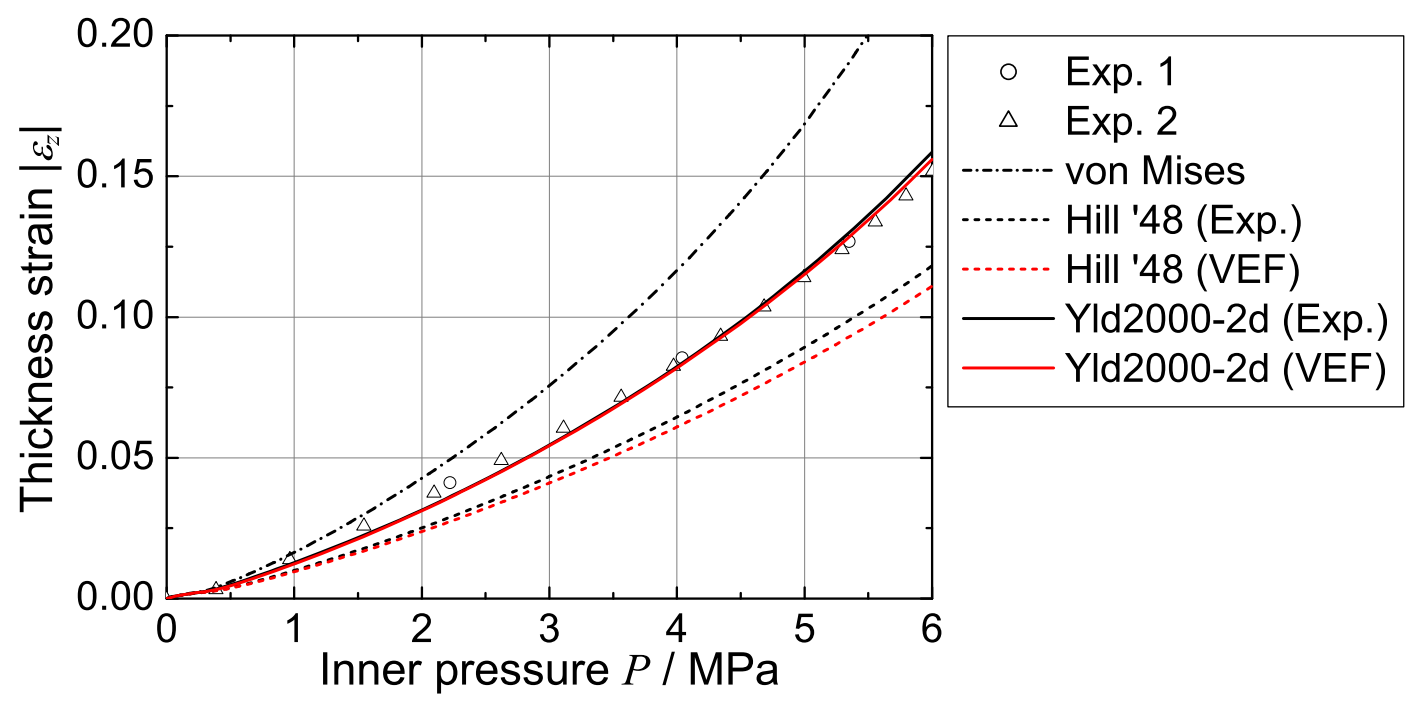

Fig. 18: Simulation bulge test: thickness strain versus inner pressure.

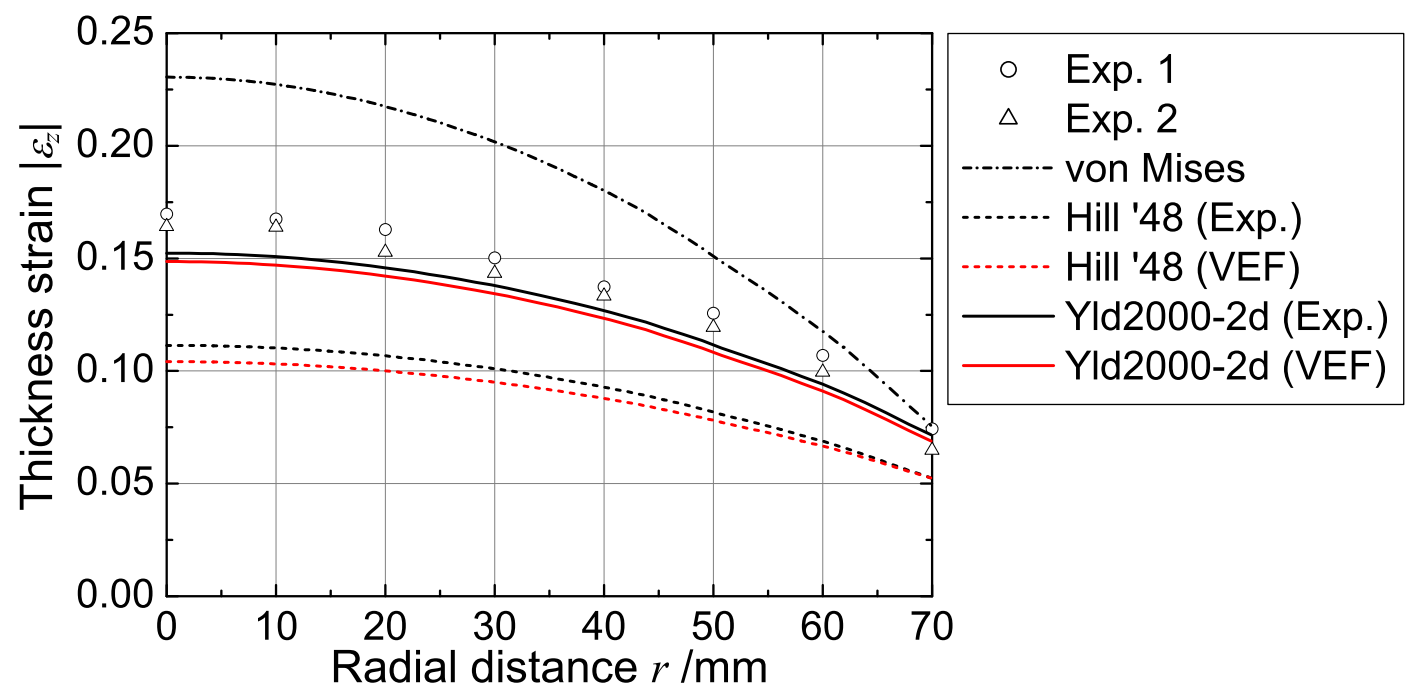

Fig. 19: Simulation bulge test: strain distribution. 


\section{Conclusions}

The following conclusions are valid for a cold-rolled low carbon steel of deep drawing quality with a nominal thickness of $1.2 \mathrm{~mm}$. Additionally, it must be noted that solely the first quadrant of stress space was probed and that the analysis was confined to the pre-necking regime of the material. Under these conditions, it is shown that the VEF has reached a sufficient level of maturity to potentially serve as a viable alternative when physical testing is impeded. Furthermore, the following can be stated:

1. The majority of the virtual data generated by the ALAMEL model is in good agreement with the physical stress-controlled tests. A somewhat weaker initial differential hardening, however, is predicted by the ALAMEL model.

2. Similar theoretical predictions of the material response (e.g. $r$-values and the direction of plastic strain rate) were obtained with either an experimental or a virtual calibration of both Hill's 48 and Yld-20002d anisotropic yield functions.

3. Equivalent FE simulation accuracy of a hydraulic bulge test was obtained using the experimentally and virtually calibrated Yld2000-2d yield function.

\section{Acknowledgements}

The authors greatly acknowledge JFE Steel Co. for providing the test material. TK gratefully acknowledges the Research Committee for the Sophistication of Steel Sheet Forming Technology with the aid of Advanced Multiaxial Stress Testing Methods established in The Iron and Steel Institute of Japan. SC is greatly indebted to R. Van Hecke (Odisee, University College Ghent, Belgium) for manufacturing the experimental set up used in section 4 . PE and AVB gratefully acknowledge financial support through the Knowledge Platform M2Form funded by the Industrial Research Fund KU Leuven.

\section{Conflict of interest}

The authors declare that they have no conflict of interest.

\section{References}

1. T. Kuwabara, T. Mori, M. Asano, T. Hakoyama, F. Barlat, Int. J. Plast. 93, 164 (2017)

2. T. Kuwabara, S. Ikeda, T. Kuroda, J. Material Process. Technol. 80/81, 517 (1998)

3. T. Kuwabara, F. Sugawara, Int. J. Plast. 45, 103 (2013)

4. F. Barlat, H. Aretz, J.W. Yoon, M.E. Karabin, J.C. Brem, R.E. Dick, Int. J. Plast. 21, 1009 (2005)

5. D. Lecompte, S. Cooreman, S. Coppieters, J. Vantomme, H. Sol, D. Debruyne, European Journal of Computational Mechanics 18:3-4, 393 (2009) 
6. A. Güner, C. Soyarslan, A. Brosius, A.E. Tekkaya, Int. J. Solids Struct. 49, 3517 (2012)

7. K. Denys, S. Coppieters, M. Seefeldt, D. Debruyne, Mechanics of Materials 100, 96 (2016)

8. M. Bertin, F. Hild, s. Roux, Strain, 52(4), 307323 (2016)

9. G. Besnard, F. Hild, S. Roux, Exp. Mech. 46(6), 789803 (2006)

10. L. Wittevrongel, D. Debruyne, S. Lomov, P. Lava, Experimental Mechanics 56 (5), 797 (2016)

11. B. Morgan, F. Hild, S. Roux, Strain (2017). DOI 10.1111/str.12233

12. F. Pierron, M. Grédiac, The Virtual Fields Method. Extracting Constitutive Mechanical Parameters from Full-field Deformation Measurements. (Springer, 2012)

13. J.H. Kim, F. Barlat, F. Pierron, M.G. Lee, Experimental Mechanics 54, 11891204 (2014)

14. M. Rossi, F. Pierron, Comput Mech 49, 5371 (2012)

15. M. Rossi, F. Pierron, Int. J. Solids Struct. 49, 420 (2012)

16. P. Lava, S. Cooreman, S. Coppieters, M. De Strycker, D. Debruyne, Optics and lasers in engineering 47 (7-8), 747 (2009)

17. J. Gawad, D. Banabic, A. Van Bael, D. Comsa, M. Gologanu, P. Eyckens, P. Van Houtte, D. Roose, Int J Plasticity. 75, 141 (2015)

18. H. Zhang, M. Diehl, R. F, D. Raabe, Int. J. Plast. 80, 111 (2016)

19. B. Plunkett, R.A. Lebesohn, O. Cazacu, F. Barlat, Acta Materialia 54, 41594169 (2006)

20. F. Grytten, B. Holmedal, O.S. Hopperstad, T. Borvik, Int. J. Plast. 12, 2248 (2008)

21. M. Kraska, M. Doig, D. Tikhomirov, R. D, F. Roters, Comp Mater Scie 46, 383 (2009)

22. K. Inal, R.K. Mishra, O. Cazacu, Int. J. Solids Struct. 47, 2223 (2010)

23. Y. An, H. Vegter, L. Carless, M. Lambriks, Int. J. Plast. 27(11), 1758 (2011)

24. A. Saai, S. Dumoullin, O.S. Hopperstad, O.G. Lademo, Comp Mater Scie 67, 424433 (2013)

25. A. Yamanaka, K. Hashimoto, J. Kawaguchi, T. Sakurai, T. Kuwabara, J. JILM 65, 561 (2015)

26. K. Zhang, B. Holmedal, O.S. Hopperstad, S. Dumoulin, J. Gawad, A. Van Bael, P. Van Houtte, Int. J. Plast. 66, 3 (2015)

27. W. Hammami, G. Gilles, A.M. Habraken, Duchêne, Int J Mater Form 4, 205:2015 (2011)

28. S. Coppieters, T. Kuwabara, Experimental Mechanics 54, 1355 (2014)

29. H. Nakano, T. Hakoyama, T. Kuwabara, in The 20th International ESAFORM Conference on Material Forming, Dublin, Ireland, 26-28 April, 2017 (2017)

30. ISO 16842. metallic materials - sheet and strip - biaxial tensile testing using a cruciform test piece (2014)

31. Y. Hanabusa, H. Takizawa, T. Kuwabara, Steel Res. Int. 81 (9), 1376 (2010)

32. Y. Hanabusa, H. Takizawa, T. Kuwabara, J. Mater. Process. Technol. 213, 961 (2013)

33. T. Kuwabara, K. Yoshida, K. Narihara, S. Takahashi, Int. J. Plasticity 21, 101 (2005)

34. P. Van Houtte, S. Li, M. Seefeldt, L. Delannay, Int J Plasticity 21(3), 589 (2005)

35. P. Eyckens, H. Mulder, J. Gawad, H. Vegter, D. Roose, T. van den Boogaard, A. Van Bael, P. Van Houtte, Int J Plasticity 73, 119 (2015)

36. L.S. Tóth, P. Van Houtte, Textures and Microstructures 19, 229 (1992)

37. R. Hill, J.W. Hutchinson, J. Appl. Mech. 59, S1 (1992)

38. R. Hill, S. Hecker, M. Stout, Int. J. Solids Struct. 31, 2999 (1994)

39. R. Von Mises, Mechanik der festen Korper un plastich deformablen Zustant. Gottingen Nachrichten (Math. Phys. Kl., 1913)

40. R. Hill, Proc. R. Soc. Lond. A193 (1033), 281 (1948)

41. F. Barlat, J.C. Brem, J.W. Yoon, K. Chung, R.E. Dick, D.J. Lege, F. Pourboghrat, S.H. Choi, E. Chu, Int. J. Plast. 19, 1297 (2003)

42. J.W. Yoon, F. Barlat, R.E. Dick, K. Chung, T.J. Kang, Int. J. Plast. 20, 495 (2004)

43. K. Ichikawa, T. Kuwabara, S. Coppieters, Key Engineering Materials 611-612, 56 (2014)

44. D. Jocham, R. Norz, W. Volk, Int. J. Mater. Form 10, 453 (2017)

45. http://matchidmbc.be/

46. Abaqus/standard 6.13 
Table A.1: Two-stage Voce hardening parameters for the material under investigation.

\begin{tabular}{llllll}
\hline Independent parameters & $\tau_{0}[\mathrm{MPa}]$ & $\tau_{\text {sat }}^{\text {III }}[\mathrm{MPa}]$ & $\tau_{\text {sat }}^{I V}[\mathrm{MPa}]$ & $\theta_{0}[\mathrm{MPa}]$ & $\theta_{T}[\mathrm{MPa}]$ \\
& 57.4 & 135.8 & 165.0 & 381.7 & 105.6 \\
\hline \multirow{2}{*}{ Dependent parameters } & $\tau_{T}[\mathrm{MPa}]$ & $\gamma_{T}[\backslash]$ & & \\
& 114.1 & 0.264 & & \\
\hline
\end{tabular}

\section{A The two-stage Voce hardening law}

The classical Voce hardening law assumes that the hardening rate $\theta=\frac{d \tau}{d \gamma}$ decreases linearly as a function of $\tau$. The currently adopted two-stage Voce law is more generic, assuming two subsequent hardening stages, denoted stages III and IV, each with a different linearly decreasing hardening rate. In $\tau-\theta$-diagram, the two-stage hardening behavior shows 2 linear parts, connected to each other at a so-called transition point. The two-stage Voce law is given by:

$$
\tau= \begin{cases}\tau_{\text {sat }}^{I I I}-\left(\tau_{\text {sat }}^{I I I}-\tau_{0}\right) \exp \left(\frac{-\theta_{0}}{\tau_{\text {sat }}^{I I I}-\tau_{0}} \gamma\right) & \text { for } \gamma \leq \gamma_{T} \\ \tau_{\text {sat }}^{I V}-\left(\tau_{\text {sat }}^{I V}-\tau_{T}\right) \exp \left(\frac{-\theta_{T}}{\tau_{\text {sat }}^{I V}-\tau_{T}}\left(\gamma-\gamma_{T}\right)\right) & \text { for } \gamma \geq \gamma_{T}\end{cases}
$$

It has 5 independent parameters, the physical meaning of which is as follows:

1. $\tau_{0}$ is the initial flow stress (i.e. of the stage III),

2. $\tau_{\text {sat }}^{I I I}$ is the saturation flow stress associated to stage III,

3. $\tau_{\text {sat }}^{I V}$ is the saturation flow stress associated to stage IV,

4. $\theta_{0}$ is the initial hardening rate (i.e. of the stage III), and

5. $\theta_{T}$ is the hardening rate of the transition point between stages III and IV.

These parameters are subject to the constraints $0<\tau_{0}<\tau_{\text {sat }}^{I I I} \leq \tau_{\text {sat }}^{I V}$ and $0<\theta_{T}<\theta_{0}$. The one-stage Voce variant is retrieved by imposing $\tau_{\text {sat }}^{I I I}=\tau_{\text {sat }}^{I V}$ and setting $\theta_{T}$ to an arbitrary value within constraint boundaries. The two-stage Voce hardening law Furthermore, $\gamma_{T}$ and $\tau_{T}$ are two derived (dependent) Voce parameters that denote the accumulated slip and the flow stress at the transition point, respectively. For the current material under investigation, Figure A-1 shows the quality of fit of the two-stage Voce law, while the corresponding Voce parameters are listed in Table A.1.

\section{B Anisotropic yield functions}

\section{B.1 Hill 1948}

Under plane stress conditions Hill's 48 yield criterion is given as

$$
F \sigma_{22}^{2}+G \sigma_{11}^{2}+H\left(\sigma_{11}-\sigma_{22}\right)^{2}+2 N \sigma_{12}^{2}=\bar{\sigma}^{2}
$$

with $\sigma_{i j}$ the Cauchy stress components with respect to the orthotropic axes. The anisotropic parameters $F, G, H$ and $N$ can be determined using the Lankford ratios $r_{\alpha}$ along three different tensile directions:

$$
\left\{\begin{array}{l}
r_{0}=\frac{H}{G}=\frac{H}{1-H} \\
r_{45}=\frac{2 N-F-G}{2(G+F)}=\frac{2 N-F+H-1}{2(1-H+F)} \\
r_{90}=\frac{H}{F}
\end{array}\right.
$$




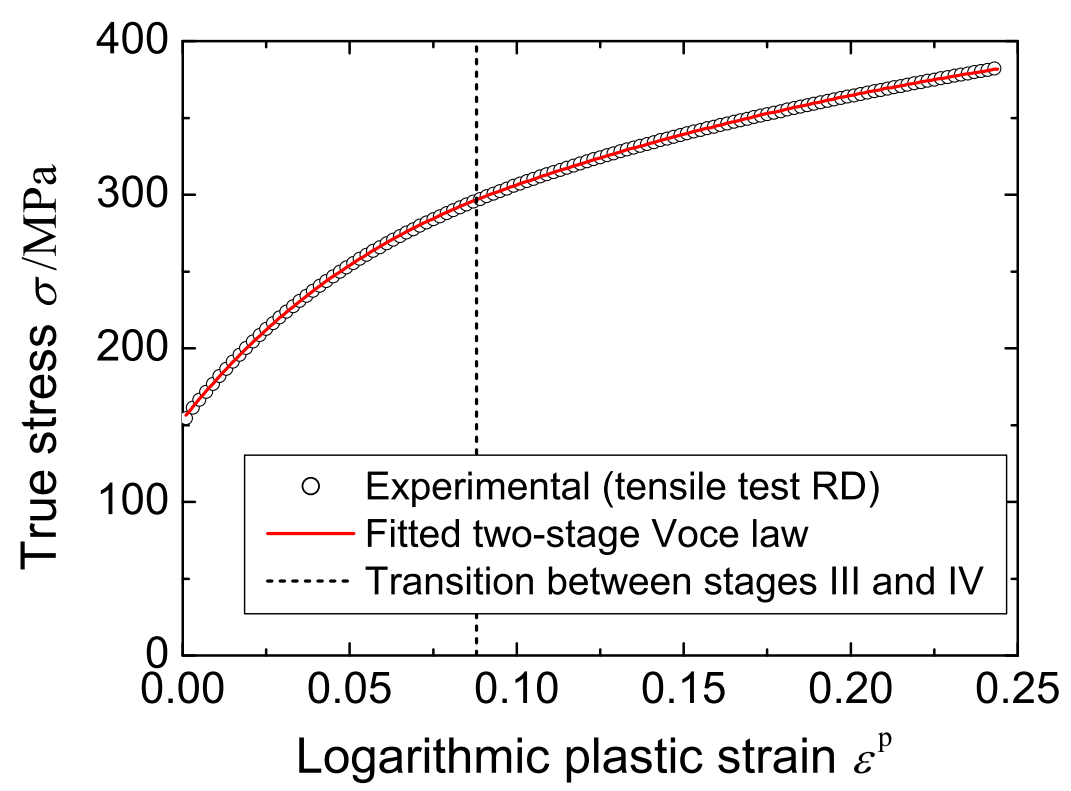

Fig. A.1: Two-stage Voce strain hardening: fitting result.

\section{B.2 Yld2000-2d}

The Yld2000-2d yield function [41] is defined as

$$
\left|X_{1}^{\prime}-X_{2}^{\prime}\right|^{M}+\left|2 X_{2}^{\prime \prime}+X_{1}^{\prime \prime}\right|^{M}+\left|2 X_{1}^{\prime \prime}+X_{2}^{\prime \prime}\right|^{M}=2 \bar{\sigma}^{M}
$$

with $\bar{\sigma}$ the equivalent stress. The principal values $X_{i}^{\prime}$ and $X_{i}^{\prime \prime}$ of the second order tensors $\mathbf{X}^{\prime}$ and $\mathbf{X}^{\prime \prime}$ are given as

$$
X_{i}^{\prime}=\frac{1}{2}\left(X_{x x}^{\prime}+X_{y y}^{\prime} \pm \sqrt{\left(X_{x x}^{\prime}-X_{y y}^{\prime}\right)^{2}+4 X_{x y}^{\prime 2}}\right)
$$

and

$$
X_{i}^{\prime \prime}=\frac{1}{2}\left(X_{x x}^{\prime \prime}+X_{y y}^{\prime \prime} \pm \sqrt{\left(X_{x x}^{\prime \prime}-X_{y y}^{\prime \prime}\right)^{2}+4 X_{x y}^{\prime \prime 2}}\right)
$$

The second order tensors $\mathbf{X}^{\prime}$ and $\mathbf{X}^{\prime \prime}$ are the result of a linear transformation of the Cauchy stress $\sigma$ :

$$
\begin{aligned}
& {\left[\begin{array}{l}
X_{11}^{\prime} \\
X_{22}^{\prime} \\
X_{12}^{\prime}
\end{array}\right]=\left[\begin{array}{ccc}
L_{11}^{\prime} & L_{12}^{\prime} & 0 \\
L_{21}^{\prime} & L_{22}^{\prime} & 0 \\
0 & 0 & L_{66}^{\prime}
\end{array}\right]\left[\begin{array}{l}
\sigma_{11} \\
\sigma_{22} \\
\sigma_{12}
\end{array}\right],\left[\begin{array}{l}
X_{1,}^{\prime \prime} \\
X_{22}^{\prime \prime} \\
X_{12}^{\prime \prime}
\end{array}\right]=\left[\begin{array}{ccc}
L_{1,1}^{\prime \prime} & L_{1,2}^{\prime \prime} & 0 \\
L_{21}^{\prime \prime} & L_{22}^{\prime \prime} & 0 \\
0 & 0 & L_{66}^{\prime \prime}
\end{array}\right]\left[\begin{array}{l}
\sigma_{11} \\
\sigma_{22} \\
\sigma_{12}
\end{array}\right],} \\
& {\left[\begin{array}{l}
L_{11}^{\prime} \\
L_{12}^{\prime} \\
L_{21}^{\prime} \\
L_{22}^{\prime} \\
L_{66}^{\prime}
\end{array}\right]=\left[\begin{array}{ccc}
\frac{2}{3} & 0 & 0 \\
-\frac{1}{3} & 0 & 0 \\
0 & -\frac{1}{3} & 0 \\
0 & \frac{2}{3} & 0 \\
0 & 0 & 1
\end{array}\right]\left[\begin{array}{l}
\alpha_{1} \\
\alpha_{2} \\
\alpha_{7}
\end{array}\right],\left[\begin{array}{l}
L_{1,1}^{\prime \prime} \\
L_{1,}^{\prime \prime} \\
L_{21}^{\prime \prime} \\
L_{22}^{\prime \prime} \\
L_{66}^{\prime \prime}
\end{array}\right]=\frac{1}{9}\left[\begin{array}{ccccc}
-2 & 2 & 8 & -2 & 0 \\
1 & -4 & -4 & 4 & 0 \\
4 & -4 & -4 & 1 & 0 \\
-2 & 8 & 2 & -2 & 0 \\
0 & 0 & 0 & 0 & 9
\end{array}\right]\left[\begin{array}{l}
\alpha_{3} \\
\alpha_{4} \\
\alpha_{5} \\
\alpha_{6} \\
\alpha_{7} \\
\alpha_{8}
\end{array}\right],}
\end{aligned}
$$

with $\sigma_{i j}$ and $\alpha_{i}$ (i=1-8) the Cauchy stress components with respect to the orthotropic axes and the anisotropic parameters, respectively. 